\title{
RESULTS OF SOME COMPRESSION TESTS OF STRUCTURAL STEEL ANGLES.
}

\author{
By A. H. Stang and L. R. Strickenberg.
}

ABSTRACT.

This article presents the results of compression tests of I 70 structural angles, made at the Pittsburgh branch, Bureau of Standards. The object of the tests was to determine the ultimate compressive strength of angles fastened at the ends in such ways as would closely correspond to their connections in the construction of transmission towers. There was also tested a series of angles with square ends. An end fixation factor was found to represent satisfactorily the effect of different types of end connections. Using this fixation factor, the average values for large slenderness ratios were well represented by Euler's formula. The results obtained from shorter columns agreed with the experimental and theoretical results of Kármán. The effect of eccentric loading was most marked at the slenderness ratios indicated by Kármán's theory.

CONTENTS.

Page.

I. Introduction.

$65 \mathrm{I}$

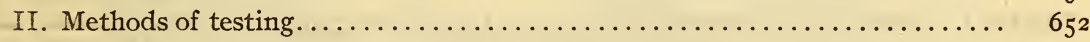

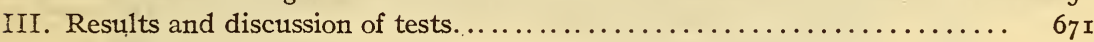

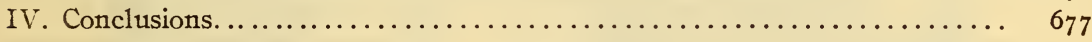

\section{INTRODUCTION.}

Compression tests of $\mathrm{I} 70$ standard rolled structural steel angles were made at the Pittsburgh laboratory of the Bureau of Standards during the spring of I 9 I 7 . The specimens tested were all furnished by the tower department of the American Bridge Co., which cooperated in planning the investigation and in carrying out the tests. As the angles were intended for legs and lattice members in electrical transmission tower construction, the greater number were tested with bolted ends, the bolting imitating the riveting used in the construction of the towers. For comparison a number of angles were also tested with flat ends. 


\section{METHOD OF TESTING.}

The specimens were all tested in a 600,000-pound Olsen testing machine. (Fig. I shows a general view of the testing machine with an angle under load.) In order to determine the deformation that took place in the angle as the load was applied, 'a special compressometer was used which was so located as to measure the shortening of the centroidal axis of the specimen. A view of the compressometer attached to a test specimen is shown in Figure 2.

The angles with square ends, having no bolts, were mounted directly between the base and the straining head of the testing machine. In order to test the bolted specimens, special fixtures of structural material were bolted to the base and straining head of the machine and the specimens bolted to these, as shown in Figure 2, a view of the connection used for two bolts in one leg of the angle. A specimen with ends folded is shown in Fig. 3.

The dimensions of the angles are shown in Tables $\mathrm{I}$ to 7 , inclusive, and in Figures 4 to 15 accompanying them. The physical and chemical properties of the material in the angles, obtained incompletely from the mill test reports, are given in Table 8 .

TABLE 1.-Results of Compression Tests on Angles with Square Ends, No Bolts.

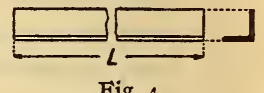

Fig. 4.

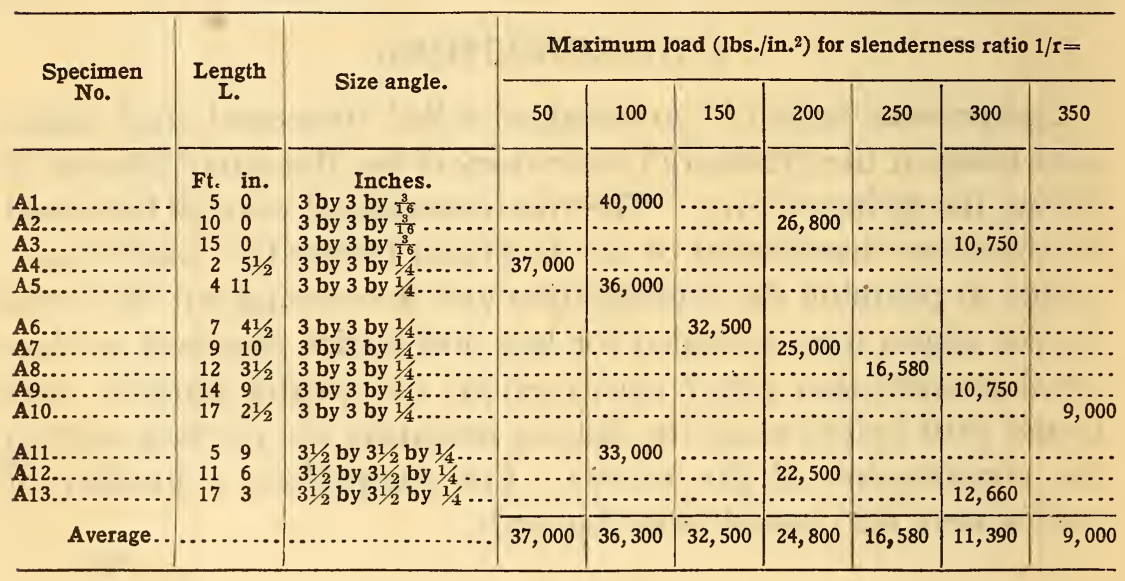


Technologic Papers of the Bureau of Standards, Vol. 16.

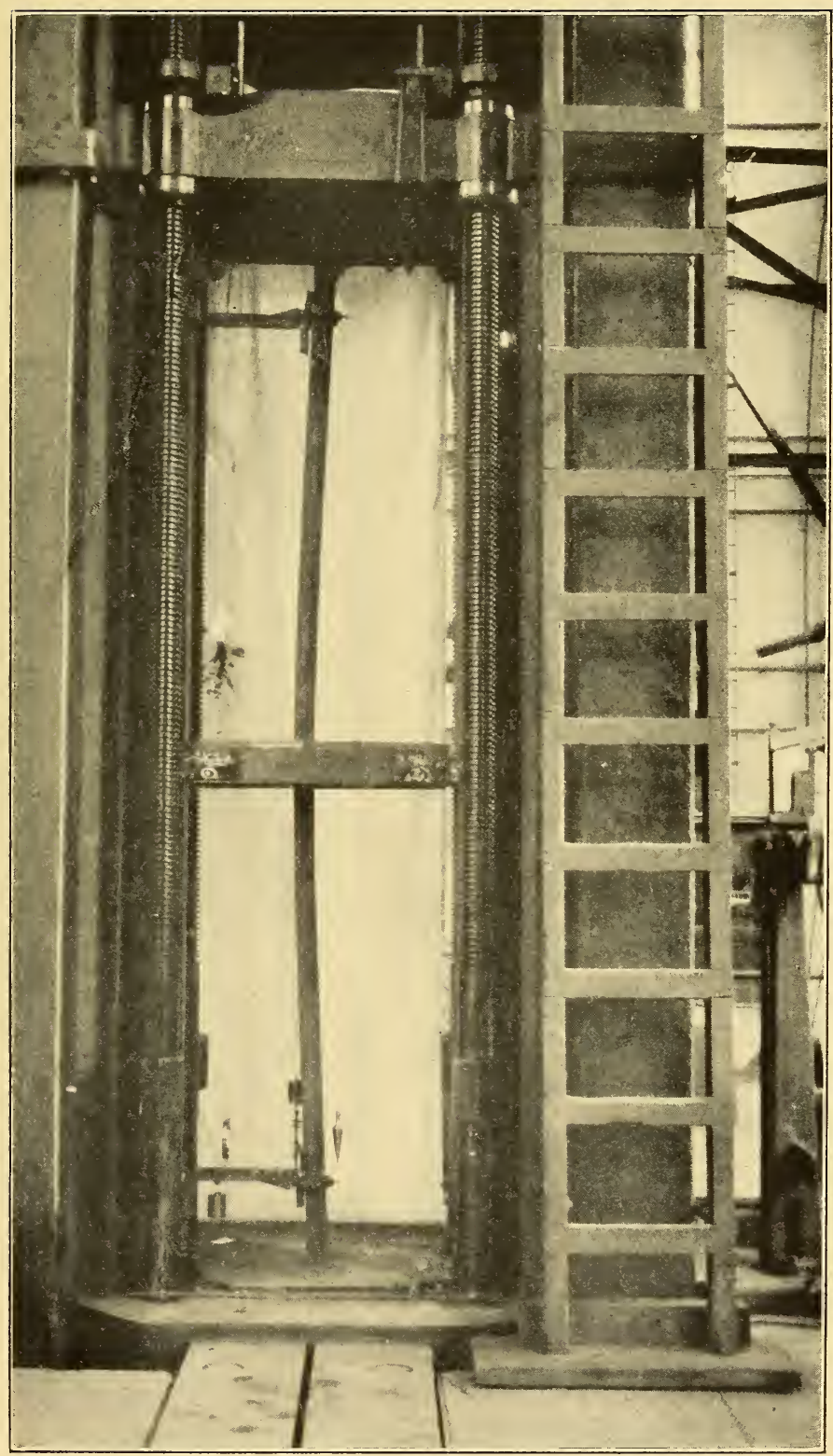

FIG. I.-View of the testing machine with an angle under load. 
Technologic Papers of the Bureau of Standards, Vol. 16.

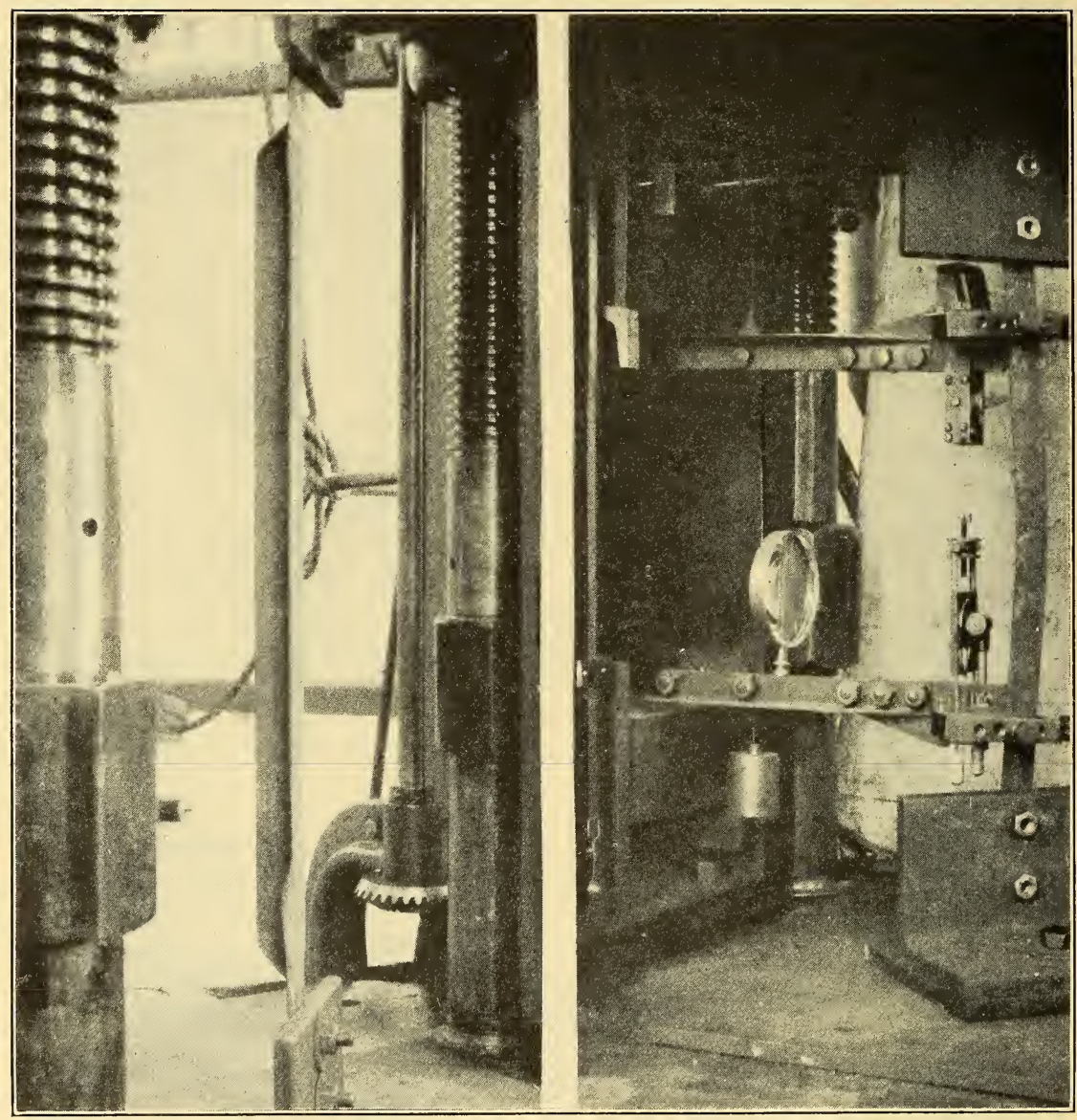

FIG. 3.-An angle with ends folded, in the testing machine.
FIG. 2.-View of compressometer and connections used for fastening a test specimen to the testing machine, two bolts in one leg. 
TABLE 2.-Results of Compression Tests on Angles with One Bolt Connection, Plain Ends.

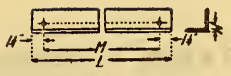

Fig. 5.

\begin{tabular}{|c|c|c|c|c|c|c|c|c|c|}
\hline \multirow{2}{*}{$\begin{array}{c}\text { Specimen } \\
\text { No. } \\
.\end{array}$} & \multirow{2}{*}{$\begin{array}{c}\text { Length } \\
\text { L. }\end{array}$} & \multirow{2}{*}{$\begin{array}{c}\text { Dis- } \\
\text { tance. } \\
\text { M. }\end{array}$} & \multirow{2}{*}{ Size angles. } & \multirow{2}{*}{$\begin{array}{c}\text { Dis- } \\
\text { tance. } \\
\text { N. }\end{array}$} & \multirow{2}{*}{$\begin{array}{l}\text { Diam- } \\
\text { eter of } \\
\text { holes. }\end{array}$} & \multicolumn{4}{|c|}{$\begin{array}{l}\text { Maximum load (lbs./in. }{ }^{2} \text { ) for } \\
\text { slenderness ratio } 1 / \mathrm{r}=\end{array}$} \\
\hline & & & & & & 200 & 250 & 300 & 350 \\
\hline 38. & $\begin{array}{rl}\text { Ft. } & \text { in. } \\
4 & 41 / 2 \\
6 & 51 / 2 \\
4 & 21 / 2 \\
6 & 21 / 2 \\
5 & 21 / 2\end{array}$ & $\begin{array}{rl}\text { Ft. } & \text { in. } \\
4 & 2 \\
6 & 3 \\
4 & 0 \\
6 & 0 \\
5 & 0\end{array}$ & $\begin{array}{l}\text { Inches. } \\
11 / 4 \text { by } 11 / 4 \text { by } \\
11 / 4 \text { by } 11 / 4 \text { by } \\
11 / 4 \text { by } 11 / 4 \text { by } \\
11 / 4 \text { by } 11 / 4 \text { by } \\
11 / 2 \text { by } 11 / 2 \text { by }\end{array}$ & $\begin{array}{r}\text { Inches. } \\
5 / 8 \\
5 / 8 \\
5 / 8 \\
5 / 8 \\
\frac{13}{16}\end{array}$ & $\begin{array}{c}\text { Inch. } \\
\frac{9}{16} \\
\frac{9}{16} \\
\frac{19}{16} \\
\frac{1}{16} \\
\frac{16}{16}\end{array}$ & $\begin{array}{r}9,070 \\
10,710 \\
\cdots \\
8,500\end{array}$ & & $\begin{array}{r}5,380 \\
5,250\end{array}$ & \\
\hline B 13 & $\begin{array}{ll}6 & 51 / 2 \\
7 & 81 / 2 \\
5 & 01 / 2 \\
7 & 51 / 2 \\
8 & 61 / 2\end{array}$ & $\begin{array}{rr}6 & 3 \\
7 & 6 \\
4 & 10 \\
7 & 3 \\
8 & 4\end{array}$ & $\begin{array}{l}11 / 2 \text { by } 11 / 2 \text { by } \\
11 / 2 \text { by } 11 / 2 \text { by } \\
11 / 2 \text { by } 11 / 2 \text { by } \\
11 / 2 \text { by } 11 / 2 \text { by } \\
2 \text { by } 2 \text { by } 1 / 8 .\end{array}$ & $\begin{array}{c}\frac{13}{16} \\
\frac{13}{16} \\
\frac{13}{16} \\
1 \frac{15}{16} \\
1\end{array}$ & $\begin{array}{l}\frac{11}{16} \\
\frac{11}{16} \\
\frac{11}{16} \\
\frac{11}{16} \\
\frac{11}{16}\end{array}$ & $\begin{array}{c}10 \\
\ldots\end{array}$ & 5,210 & \begin{tabular}{r}
4,660 \\
\hdashline 5,950 \\
$\ldots \ldots \ldots$
\end{tabular} & \\
\hline B20. & $\begin{array}{rr}10 & 21 / 2 \\
8 & 61 / 2 \\
10 & 21 / 2 \\
10 & 71 / 2 \\
12 & 81 / 2\end{array}$ & $\begin{array}{rl}10 & 0 \\
8 & 4 \\
10 & 0 \\
10 & 5 \\
12 & 6\end{array}$ & $\begin{array}{l}2 \text { by } \\
2 \text { by } \\
2 \text { by } \\
21 / 2 b \\
21 / 2 b\end{array}$ & $\begin{array}{l}1 \\
1 \\
1 \\
11 / 4 \\
11 / 4\end{array}$ & $\begin{array}{l}\frac{11}{16} \\
\frac{11}{16} \\
\frac{11}{16} \\
\frac{11}{16} \\
\frac{11}{16}\end{array}$ & & $\begin{array}{r}5,500 \\
\quad 5,500 \\
\ldots \ldots \ldots\end{array}$ & $\begin{array}{r}4,000 \\
\cdots \\
4,450 \\
\cdots \\
3,350\end{array}$ & \\
\hline B21. & $\begin{array}{ll}14 & 91 / 2 \\
12 & 51 / 2 \\
10 & 5 \\
12 & 51 / 2 \\
14 & 6\end{array}$ & $\begin{array}{ll}14 & 7 \\
12 & 3 \\
10 & 21 / 2 \\
12 & 3 \\
14 & 31 / 2\end{array}$ & $\begin{array}{l}21 / 2 b \\
21 / 2 b \\
21 / 2 b \\
21 / 2 b \\
21 / 2 b\end{array}$ & $\begin{array}{l}11 / 4 \\
11 / 4 \\
11 / 4 \\
11 / 4 \\
11 / 4\end{array}$ & $\begin{array}{l}\frac{11}{16} \\
\frac{11}{16} \\
\frac{11}{16} \\
\frac{11}{16} \\
\frac{11}{16}\end{array}$ & & $\begin{array}{r}6,000 \\
\ldots \ldots \ldots\end{array}$ & $\begin{array}{r}4,000 \\
\cdots 4,760\end{array}$ & $\begin{array}{c}2,610 \\
\ldots \ldots . .\end{array}$ \\
\hline $\begin{array}{l}\text { B26 } \\
\text { B27 } \\
\text { B28 } \\
\text { B29 } \\
\text { B29 }\end{array}$ & $\begin{array}{ll}12 & 81 / 2 \\
15 & 21 / 2 \\
17 & 81 / 2 \\
15 & 21 / 2 \\
15 & 21 / 2\end{array}$ & $\begin{array}{ll}12 & 6 \\
15 & 0 \\
17 & 6 \\
15 & 0 \\
15 & 0\end{array}$ & 3 by & $\begin{array}{l}11 / 2 \\
11 / 2 \\
11 / 2 \\
11 / 2 \\
11 / 2\end{array}$ & $\begin{array}{l}\frac{11}{16} \\
\frac{11}{16} \\
\frac{11}{16} \\
\frac{11}{18} \\
\frac{18}{16}\end{array}$ & & 4,670 & $\begin{array}{r}3,150 \\
\dddot{3}, 890 \\
3,880\end{array}$ & 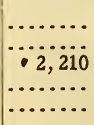 \\
\hline $\begin{array}{l}\text { B30.... } \\
\text { B30A... } \\
\text { BX30.. } \\
\text { B31 } \\
\text { BX31.. }\end{array}$ & $\begin{array}{ll}12 & 6 \\
12 & 6 \\
12 & 6 \\
14 & 111 / 2 \\
14 & 111 / 2\end{array}$ & $\begin{array}{ll}12 & 31 / 2 \\
12 & 31 / 2 \\
12 & 31 / 2 \\
14 & 9 \\
14 & 9\end{array}$ & $\begin{array}{l}3 \text { by } 3 \text { by } 1 / 4 \ldots \\
3 \text { by } 3 \text { by } 1 / 4 \ldots \\
3 \text { by } 3 \text { by } 1 / 4 \cdots \\
3 \text { by } 3 \text { by } 1 / 4 \ldots \\
3 \text { by } 3 \text { by } 1 / 4 \ldots\end{array}$ & $\begin{array}{l}11 / 2 \\
11 / 2 \\
11 / 2 \\
11 / 2 \\
11 / 2\end{array}$ & $\begin{array}{l}\frac{11}{16} \\
\frac{11}{16} \\
\frac{11}{16} \\
\frac{16}{16} \\
\frac{11}{16}\end{array}$ & & $\begin{array}{l}5,500 \\
5,400 \\
5,420\end{array}$ & $\begin{array}{r}3,930 \\
4,450\end{array}$ & \\
\hline $\begin{array}{l}\text { B32... } \\
\text { BX32. } \\
\text { B33... } \\
\text { B34... } \\
\text { B35... }\end{array}$ & $\begin{array}{ll}17 & 5 \\
17 & 5 \\
17 & 51 / 2 \\
20 & 4 \\
17 & 51 / 2\end{array}$ & $\begin{array}{ll}17 & 21 / 2 \\
17 & 21 / 2 \\
17 & 3 \\
20 & 11 / 2 \\
17 & 3\end{array}$ & $\begin{array}{l}3 \text { by } \\
3 \text { by } \\
31 / 21 \\
31 / 21 \\
31 / 2\end{array}$ & $\begin{array}{l}11 / 2 \\
11 / 2 \\
11 / 2 \\
11 / 2 \\
11 / 2\end{array}$ & $\begin{array}{l}\frac{12}{16} \\
\frac{11}{16} \\
\frac{18}{16} \\
\frac{11}{18} \\
\frac{11}{16}\end{array}$ & & & $\begin{array}{r}4,000 \\
3,540\end{array}$ & \begin{tabular}{r}
2,750 \\
3,270 \\
\hdashline, 920 \\
2,920
\end{tabular} \\
\hline 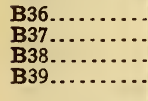 & $\begin{array}{ll}20 & 4 \\
11 & 51 / 2 \\
13 & 81 / 2 \\
15 & 111 / 2\end{array}$ & $\begin{array}{ll}20 & 11 / 2 \\
11 & 3 \\
13 & 6 \\
15 & 9\end{array}$ & $\begin{array}{l}31 / 2 \text { by } 31 / 2 \text { by } 1 / 4 \ldots \\
31 / 2 \text { by } 21 / 2 \text { by } 1 / 4 \ldots \\
31 / 2 \text { by } 21 / 2 \text { by } 1 / 4 \ldots \\
31 / 2 \text { by } 21 / 2 \text { by } 1 / 4 \ldots\end{array}$ & $\begin{array}{l}11 / 2 \\
11 / 4 \\
11 / 4 \\
11 / 4\end{array}$ & $\begin{array}{l}\frac{11}{16} \\
\frac{11}{16} \\
\frac{11}{16} \\
\frac{11}{16}\end{array}$ & & $\begin{array}{c}5,000 \\
\cdots \cdots \\
\cdots \cdots\end{array}$ & $\begin{array}{r}3,410 \\
\cdots\end{array}$ & \begin{tabular}{r}
2,720 \\
\hdashline$\ldots .$. \\
$\dddot{3}, 500$
\end{tabular} \\
\hline & & & & & & 9,610 & 5,460 & 4,240 & 2,91 \\
\hline
\end{tabular}

TABLE 3.-Results of Compression Tests on Angles with One Bolt Connection in One Leg Only, Ends Folded.

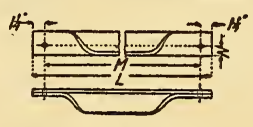

Fig. 6.

\begin{tabular}{|c|c|c|c|c|c|c|c|c|}
\hline \multirow{2}{*}{ Specimen No. } & \multirow{2}{*}{$\begin{array}{l}\text { Length } \\
\text { L. }\end{array}$} & \multirow{2}{*}{$\begin{array}{c}\text { Distance } \\
\text { M. }\end{array}$} & \multirow{2}{*}{ Size angle. } & \multirow{2}{*}{$\begin{array}{l}\text { Dis- } \\
\text { tance } \\
\text { IN. }\end{array}$} & \multirow{2}{*}{$\begin{array}{l}\text { Diam- } \\
\text { eter of } \\
\text { holes. }\end{array}$} & \multicolumn{3}{|c|}{$\begin{array}{l}\text { Maximum load (lbs./ } \\
\text { in. }{ }^{2} \text { ) for slenderness } \\
\text { ratio } 1 / \mathrm{r}=\end{array}$} \\
\hline & & & & & & 100 & 200 & 300 \\
\hline \multirow[t]{2}{*}{ 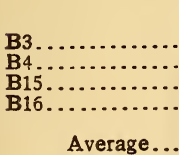 } & \multirow[t]{2}{*}{$\begin{array}{rr}\text { Ft. } & \text { in. } \\
2 & 31 / 2 \\
4 & 41 / 2 \\
6 & 101 / 2 \\
10 & 21 / 2\end{array}$} & \multirow[t]{2}{*}{$\begin{array}{rr}\text { Ft. } & \text { in. } \\
2 & 1 \\
4 & 2 \\
6 & 8 \\
10 & 0\end{array}$} & \multirow[t]{2}{*}{$\begin{array}{c}\text { Inches. } \\
11 / 4 \text { by } 11 / 4 \text { by } 1 / 8 . \\
11 / 4 \text { by } 11 / 4 \text { by } 1 / 8 . \\
2 \text { by } 2 \text { by } 1 / 8 \ldots \ldots \\
2 \text { by } 2 \text { by } 1 / 8 \ldots \ldots\end{array}$} & \multirow[t]{2}{*}{$\begin{array}{c}\text { Inch. } \\
\frac{5}{5} \\
1 \\
1\end{array}$} & \multirow[t]{2}{*}{$\begin{array}{r}\text { Inch. } \\
\frac{9}{16} \\
\frac{9}{16} \\
\frac{11}{16} \\
\frac{11}{16}\end{array}$} & $\begin{array}{l}14,900 \\
\cdots \cdots \cdots \\
\cdots \cdots \\
\cdots \cdots\end{array}$ & $\begin{array}{l}9250 \\
7400\end{array}$ & 4500 \\
\hline & & & & & & 14,900 & 8325 & 4500 \\
\hline
\end{tabular}


TABLE 4.-Results of Compression Tests on Angles with Two Bolt Connections, One Leg Only, Plain Ends.

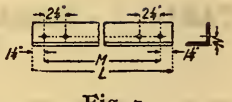

Fig. 7 .

\begin{tabular}{|c|c|c|c|c|c|c|c|c|c|}
\hline \multirow{2}{*}{$\begin{array}{l}\text { Specimen } \\
\text { No. }\end{array}$} & \multirow{2}{*}{$\begin{array}{c}\text { Length } \\
\text { L. }\end{array}$} & \multirow{2}{*}{$\begin{array}{l}\text { Dis- } \\
\text { tance. } \\
\text { M. }\end{array}$} & \multirow{2}{*}{ Size angles. } & \multirow{2}{*}{$\begin{array}{l}\text { Dis- } \\
\text { tance. } \\
\text { N. }\end{array}$} & \multirow{2}{*}{$\begin{array}{l}\text { Diam- } \\
\text { eter of } \\
\text { holes. }\end{array}$} & \multicolumn{4}{|c|}{$\begin{array}{l}\text { Maximum load (lbs./in. } .^{2} \text { ) for } \\
\text { slenderness ratio } 1 / r=\end{array}$} \\
\hline & & & & & & 100 & 150 & 200 & 250 \\
\hline $\begin{array}{l}\text { C1... } \\
\text { C2... } \\
\text { C3... } \\
\text { C3a.. } \\
\text { C4... }\end{array}$ & $\begin{array}{rr}\text { Ft. } & \text { in. } \\
2 & 53 / 4 \\
2 & 43 / 4 \\
2 & 103 / 4 \\
2 & 103 / 4 \\
4 & 13 / 4\end{array}$ & $\begin{array}{rr}\text { Ft. in. } \\
1 & 103 / 4 \\
1 & 93 / 4 \\
2 & 33 / 4 \\
2 & 33 / 4 \\
3 & 63 / 4\end{array}$ & $\begin{array}{c}\text { Inches. } \\
11 / 4 \text { by } 11 / 4 \text { by } 1 / 8 \ldots \\
11 / 4 \text { by } 11 / 4 \text { by } \frac{3}{16} \ldots \\
11 / 2 \text { by } 11 / 2 \text { by } 1 / 8 \\
11 / 2 \text { by } 11 / 2 \text { by } 1 / 8 \\
11 / 2 \text { by } 11 / 2 \text { by } 1 / 8 \ldots\end{array}$ & $\begin{array}{r}\text { Inches. } \\
5 / 8 \\
5 / 8 \\
13 \\
16 \\
\frac{13}{16} \\
\frac{16}{15} \\
16\end{array}$ & $\begin{array}{r}\text { Inch. } \\
\frac{9}{16} \\
\frac{9}{16} \\
11 \\
16 \\
11 \\
\frac{11}{16} \\
\frac{16}{16}\end{array}$ & $\begin{array}{r}27,200 \\
24,200 \\
23,500 \\
24,000 \\
\ldots \ldots \ldots\end{array}$ & 19,470 & $\begin{array}{l}\cdots \\
\cdots \\
\cdots \\
\cdots \\
\cdots\end{array}$ & $\begin{array}{l}\cdots \\
\cdots \\
\cdots\end{array}$ \\
\hline $\begin{array}{l}\text { C6... } \\
\text { C7... } \\
\text { C8... } \\
\text { C11.. }\end{array}$ & $\begin{array}{ll}2 & 93 / 4 \\
4 & 01 / 4 \\
5 & 43 / 4 \\
7 & 03 / 4 \\
5 & 43 / 4\end{array}$ & $\begin{array}{ll}2 & 23 / 4 \\
3 & 51 / 4 \\
4 & 93 / 4 \\
6 & 53 / 4 \\
4 & 93 / 4\end{array}$ & $\begin{array}{l}11 / 2 \text { by } 11 / 2 \text { by } \frac{3}{16} \\
11 / 2 \text { by } 11 / 2 \text { by } \frac{3}{16} \\
2 \text { by } 2 \text { by } 1 / 8 \ldots \\
2 \text { by } 2 \text { by } 1 / 8 \ldots \\
2 \text { by } 2 \text { by } \frac{3}{16} \ldots\end{array}$ & $\begin{array}{l}1^{\frac{13}{16}} \\
1 \\
1 \\
1\end{array}$ & $\begin{array}{l}11 \\
16 \\
\frac{1}{16} \\
\frac{11}{16} \\
\frac{11}{16} \\
\frac{11}{16} \\
\frac{1}{16}\end{array}$ & $\begin{array}{r}26,900 \\
\cdots \cdots \\
\cdots \cdots\end{array}$ & $\begin{array}{c}23,400 \\
15,350 \\
\cdots 18,500\end{array}$ & 14,500 & \\
\hline $\begin{array}{l}\text { C13.. } \\
\text { C14.. } \\
\text { C15.. } \\
\text { C16.. }\end{array}$ & $\begin{array}{ll}7 & 03 / 4 \\
6 & 73 / 4 \\
8 & 83 / 4 \\
6 & 61 / 4 \\
8 & 63 / 4\end{array}$ & $\begin{array}{lr}6 & 53 / 4 \\
6 & 03 / 4 \\
8 & 13 / 4 \\
5 & 11 \frac{1}{4} \\
7 & 11 \frac{3}{4}\end{array}$ & $\begin{array}{l}2 \text { by } 2 \text { by } \frac{3}{16} \ldots \ldots \\
21 / 2 \text { by } 21 / 2 \text { by } 1 / 8 \ldots \\
21 / 2 \text { by } 21 / 2 \text { by } 1 / 8 \ldots \\
21 / 2 \text { by } 21 / 2 \text { by } \frac{3}{16} \ldots \\
21 / 2 \text { by } 21 / 2 \text { by } \frac{8}{16} \ldots\end{array}$ & $\begin{array}{l}1 \\
11 / 4 \\
11 / 4 \\
11 / 4 \\
11 / 4\end{array}$ & $\begin{array}{l}\frac{11}{16} \\
\frac{11}{16} \\
\frac{11}{16} \\
\frac{11}{16} \\
\frac{11}{16}\end{array}$ & & $\begin{array}{l}13,800 \\
17,250 \\
\ldots \ldots \ldots\end{array}$ & $\begin{array}{l}14,200 \\
10,910 \\
13,830\end{array}$ & \\
\hline $\begin{array}{l}\text { C18.... } \\
\text { C19.... } \\
\text { C20.... } \\
\text { C21.... }\end{array}$ & $\begin{array}{rr}6 & 61 / 4 \\
8 & 63 / 4 \\
7 & 103 / 4 \\
10 & 43 / 4 \\
10 & 43 / 4\end{array}$ & $\begin{array}{cc}5 & 113 / 4 \\
7 & 11^{3} / 4 \\
7 & 33 / 4 \\
9 & 93 / 4 \\
9 & 93 / 4\end{array}$ & $\begin{array}{l}21 / 2 \text { by } 21 / 2 \text { by } 1 / 4 \\
21 / 2 \text { by } 21 / 2 \text { by } 1 / 4 \\
3 \text { by } 3 \text { by } 1 / 8 \ldots \\
3 \text { by } 3 \text { by } 1 / 8 \ldots \\
3 \text { by } 3 \text { by } \frac{8}{16} \ldots\end{array}$ & $\begin{array}{l}11 / 4 \\
11 / 4 \\
11 / 2 \\
11 / 2 \\
11 / 2\end{array}$ & $\begin{array}{l}\frac{11}{16} \\
\frac{11}{16} \\
1 \frac{1}{16} \\
\frac{11}{16} \\
\frac{11}{16} \\
\frac{11}{16}\end{array}$ & & $\begin{array}{c}16,995 \\
12,400 \\
\ldots \ldots \ldots \\
\ldots \ldots \ldots\end{array}$ & $\begin{array}{r}12,780 \\
\cdots 8,950 \\
12,520\end{array}$ & \\
\hline $\begin{array}{l}\text { C21a.. } \\
\text { C22... } \\
\text { C22a.. } \\
\text { C23... } \\
\text { C23a. }\end{array}$ & $\begin{array}{rl}10 & 43 / 4 \\
7 & 91 / 4 \\
7 & 91 / 4 \\
10 & 23 / 4 \\
10 & 23 / 4\end{array}$ & $\begin{array}{ll}9 & 93 / 4 \\
7 & 21 / 4 \\
7 & 21 / 4 \\
9 & 73 / 4 \\
9 & 73 / 4\end{array}$ & $\begin{array}{l}3 \text { by } 3 \text { by } 1 / 4 \ldots \ldots \\
3 \text { by } 3 \text { by } 1 / 4, \ldots \ldots \\
3 \text { by } 3 \text { by } 1 / 4 \ldots \ldots \\
3 \text { by } 3 \text { by } 1 / 4 \ldots \ldots\end{array}$ & $\begin{array}{l}11 / 2 \\
11 / 2 \\
11 / 2 \\
11 / 2 \\
11 / 2\end{array}$ & $\begin{array}{l}\frac{11}{16} \\
\frac{11}{16} \\
\frac{11}{16} \\
11 \\
116 \\
\frac{11}{16}\end{array}$ & & $\begin{array}{l}\because 15,210 \\
16,400 \\
\ldots \ldots \ldots \\
\ldots \ldots \ldots\end{array}$ & \begin{tabular}{c}
13,400 \\
\hdashline$\ldots \ldots$ \\
\hdashline 11,700 \\
12,530
\end{tabular} & \\
\hline $\begin{array}{l}\mathrm{C} 24 \ldots \ldots \ldots \ldots \\
\mathrm{C} 24 \mathrm{a} \ldots \ldots \ldots \ldots . \\
\mathrm{C} 25 \ldots \ldots \ldots \\
\mathrm{C} 25 \mathrm{a} \ldots \ldots \ldots . . \\
\mathrm{C} 26 \ldots \ldots \ldots\end{array}$ & $\begin{array}{lr}10 & 23 / 4 \\
10 & 23 / 4 \\
12 & 81 / 4 \\
12 & 81 / 4 \\
11 & 103 / 4\end{array}$ & $\begin{array}{rr}9 & 73 / 4 \\
9 & 73 / 4 \\
12 & 11 / 4 \\
12 & 11 / 4 \\
11 & 33 / 4\end{array}$ & $\begin{array}{l}3 \text { by } 3 \text { by } \frac{5}{16} \ldots \ldots \\
3 \text { by } 3 \text { by } \frac{5}{16} \ldots \ldots \\
3 \text { by } 3 \text { by } \frac{5}{16} \ldots \ldots \\
3 \text { by } 3 \text { by } \frac{5}{16} \ldots \ldots \\
31 / 2 \text { by } 31 / 2 \text { by } \frac{3}{16} \ldots\end{array}$ & $\begin{array}{l}11 / 2 \\
11 / 2 \\
11 / 2 \\
11 / 2 \\
11 / 2\end{array}$ & $\begin{array}{l}11 \\
16 \\
11 \\
16 \\
1 \frac{1}{16} \\
11 \\
\frac{11}{16} \\
\frac{11}{16}\end{array}$ & & & \begin{tabular}{c}
11,800 \\
12,570 \\
$\cdots \ldots \ldots$ \\
\hdashline 11,300
\end{tabular} & $\begin{array}{r}10,000 \\
8,350\end{array}$ \\
\hline $\begin{array}{l}\text { C27...... } \\
\text { C28...... } \\
\text { C28a..... } \\
\text { C29...... } \\
\text { C29a.... }\end{array}$ & $\begin{array}{lr}14 & 91 / 4 \\
11 & 103 / 4 \\
11 & 103 / 4 \\
14 & 91 / 4 \\
14 & 91 / 4\end{array}$ & $\begin{array}{ll}14 & 21 / 4 \\
11 & 33 / 4 \\
11 & 33 / 4 \\
14 & 21 / 4 \\
14 & 21 / 4\end{array}$ & $\begin{array}{l}31 / 2 b \\
31 / 2 b \\
31 / 2 b \\
31 / 2 b \\
31 / 2 b\end{array}$ & $\begin{array}{l}11 / 2 \\
11 / 2 \\
11 / 2 \\
11 / 2 \\
11 / 2\end{array}$ & $\begin{array}{l}\frac{11}{16} \\
1 \frac{1}{11} \\
\frac{11}{16} \\
\frac{11}{16} \\
\frac{11}{16} \\
\frac{11}{16} \\
16\end{array}$ & & & $\begin{array}{r}9,870 \\
10,400 \\
\ldots \ldots\end{array}$ & $\begin{array}{r}8,350 \\
\ldots \ldots \\
6,300 \\
7,850\end{array}$ \\
\hline $\begin{array}{l}\text { C30...... } \\
\text { C30a.... } \\
\text { C31..... }\end{array}$ & $\begin{array}{rr}14 & 91 / 4 \\
14 & 91 / 4 \\
9 & 43 / 4\end{array}$ & $\begin{array}{rr}14 & 21 / 4 \\
14 & 21 / 4 \\
8 & 93 / 4\end{array}$ & $\begin{array}{l}31 / 2 \text { by } 31 / 2 \\
31 / 2 \text { by } 31 / 2 \\
31 / 2 \text { by } 21 / 2\end{array}$ & $\begin{array}{l}11 / 2 \\
11 / 2 \\
11 / 2\end{array}$ & $\begin{array}{l}\frac{11}{16} \\
\frac{11}{16} \\
\frac{11}{16}\end{array}$ & & & 11,800 & $\begin{array}{r}8,800 \\
9,100 \\
\ldots \ldots\end{array}$ \\
\hline & & & & & & 25,200 & 16,880 & 12,050 & 8,400 \\
\hline
\end{tabular}


TABLE 5.--Results of Compression Tests on Angles with Two Bolt Connections, One Leg Only, Ends Folded.

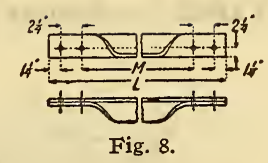

\begin{tabular}{|c|c|c|c|c|c|c|}
\hline \multirow{2}{*}{ Specimen No. } & \multirow{2}{*}{$\begin{array}{l}\text { Length } \\
\text { L. }\end{array}$} & \multirow{2}{*}{$\begin{array}{l}\text { Distance } \\
\text { M. }\end{array}$} & \multirow{2}{*}{ Size angle. } & \multirow{2}{*}{$\begin{array}{l}\text { Diameter } \\
\text { of holes. }\end{array}$} & \multicolumn{2}{|c|}{$\begin{array}{l}\text { Maximum load (lbs./ } \\
\text { in.2) for slenderness } \\
\text { ratio } 1 / \mathrm{r}=\end{array}$} \\
\hline & & & & & 50 & 100 \\
\hline $\begin{array}{l}\mathrm{C} 9 . . . \\
\mathrm{C} 10 . .\end{array}$ & $\begin{array}{rl}\text { Ft. } & \text { in. } \\
2 & 034 \\
3 & 83 / 4\end{array}$ & \begin{tabular}{rr|} 
Ft. & in. \\
1 & $53 / 4$ \\
3 & $13 / 4$
\end{tabular} & $\begin{array}{c}\text { Inches. } \\
2 \text { by } 2 \text { by } 1 / 8 \ldots \ldots \cdots \cdots \\
2 \text { by } 2 \text { by } 1 / 8 \ldots \ldots \cdots \cdots\end{array}$ & Inch. & $\begin{array}{c}16,530 \\
\ldots . . . \cdots\end{array}$ & 18,000 \\
\hline
\end{tabular}

TABLE 6.-Results of Compression Tests on Angles with Two Bolt Connections, One Bolt in Each Leg.

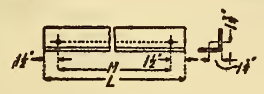

Fig. 9.

Specimens No. Dr-Dro.

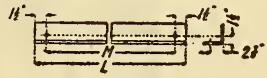

Fig. 10.

Specimens No. Er-E8.

\begin{tabular}{|c|c|c|c|c|c|c|c|c|}
\hline \multirow{2}{*}{$\begin{array}{l}\text { Specimen } \\
\text { No. }\end{array}$} & \multirow{2}{*}{$\begin{array}{l}\text { Length } \\
\text { L. }\end{array}$} & \multirow{2}{*}{$\begin{array}{l}\text { Distance } \\
\text { M. }\end{array}$} & \multirow{2}{*}{ Size angle. } & \multirow{2}{*}{$\begin{array}{l}\text { Diameter } \\
\text { of holes. }\end{array}$} & \multicolumn{4}{|c|}{$\begin{array}{c}\text { Maximum load (lbs./in. }{ }^{2} \text { ) for slenderness } \\
\text { ratio } 1 / \mathrm{r}=\end{array}$} \\
\hline & & & & & 200 & 250 & 300 & 350 \\
\hline $\begin{array}{l}\mathrm{D} 1 \ldots \ldots \\
\mathrm{D} 2 \ldots \ldots \\
\mathrm{D} 3 \ldots \ldots \\
\mathrm{D} 3 \mathrm{\ldots} \\
\mathrm{D} 4 \ldots \ldots\end{array}$ & $\begin{array}{ll}\text { Ft. } & \text { in. } \\
10 & 3 \\
12 & 9 \\
10 & 1 \\
10 & 1 \\
12 & 61 / 2\end{array}$ & $\begin{array}{rl}\text { Ft. in. } \\
10 & 0 \\
12 & 6 \\
9 & 10 \\
9 & 10 \\
12 & 31 / 2\end{array}$ & $\begin{array}{l}\text { Inches } \\
3 \text { by } 3 \text { by } \frac{3}{16} \\
3 \text { by } 3 \text { by } \frac{3}{16} \text {. } \\
3 \text { by } 3 \text { by } 1 / 4 . \\
3 \text { by } 3 \text { by } 1 / 4 . \\
3 \text { ry } 3 \text { by } 1 / 4 .\end{array}$ & 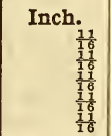 & $\begin{array}{r}13,820 \\
\ldots . \\
11,620 \\
12,400 \\
\ldots .\end{array}$ & $\begin{array}{r}10,980 \\
\cdots \ldots \ldots \ldots \\
\cdots \\
9,760\end{array}$ & (..... & $\cdots$ \\
\hline $\begin{array}{l}\text { DX } 4 \ldots \ldots \\
\text { D5 } \ldots \ldots \\
\text { DX } 5 \ldots \ldots \\
\text { D6 } \ldots \ldots \\
\text { D7 } \ldots \ldots \ldots\end{array}$ & $\begin{array}{ll}12 & 61 / 2 \\
10 & 1 \\
10 & 1 \\
11 & 9 \\
14 & 71 / 2\end{array}$ & $\begin{array}{rl}12 & 31 / 2 \\
9 & 10 \\
9 & 10 \\
11 & 6 \\
14 & 41 / 2\end{array}$ & $\begin{array}{l}3 \text { by } 3 \text { by } 1 / 4 \\
3 \text { by } 3 \text { by } \frac{5}{16} \\
3 \text { by } 3 \text { by } \frac{5}{16} \\
31 / 2 \text { by } 31 / 2 \text { b } \\
31 / 2 \text { by } 31 / 2 \text { b }\end{array}$ & $\begin{array}{l}\frac{11}{116} \\
\frac{11}{16} \\
\frac{11}{16} \\
\frac{11}{16} \\
\frac{11}{16}\end{array}$ & $\begin{array}{r}13,700 \\
13,600 \\
9,420 \\
\ldots .\end{array}$ & 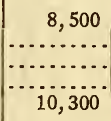 & & $\begin{array}{l}- \\
- \\
- \\
-\end{array}$ \\
\hline $\begin{array}{l}\text { D8 } \ldots \ldots \ldots \\
\text { D9 } \ldots \ldots \ldots \\
\text { D10...... } \\
\text { E1 } \ldots \ldots \ldots \\
\text { E2 } \ldots \ldots \ldots\end{array}$ & $\begin{array}{ll}17 & 6 \\
14 & 71 / 2 \\
17 & 6 \\
16 & 81 / 2 \\
20 & 0\end{array}$ & $\begin{array}{ll}17 & 3 \\
14 & 41 / 2 \\
17 & 3 \\
16 & 51 / 2 \\
19 & 9\end{array}$ & $\begin{array}{l}31 / 2 \text { by } 31 / 2 \text { by } \\
31 / 2 \text { by } 31 / 2 \text { by } \\
31 / 2 \text { by } 31 / 2 \text { by } \\
4 \text { by } 4 \text { by } 1 / 4 . \\
4 \text { by } 4 \text { by } 1 / 4 .\end{array}$ & $\begin{array}{l}\frac{11}{16} \\
\frac{1}{16} \\
16 \\
\frac{1}{16} \\
\frac{11}{16} \\
\frac{11}{16} \\
1 \frac{1}{16}\end{array}$ & & $\begin{array}{r}9,000 \\
5,960 \\
\cdots\end{array}$ & $\begin{array}{r}6,520 \\
\ldots \ldots \\
6,130 \\
4,530\end{array}$ & \\
\hline 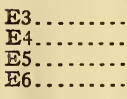 & $\begin{array}{ll}23 & 31 / 2 \\
20 & 0 \\
16 & 81 / 2 \\
20 & 0\end{array}$ & $\begin{array}{ll}23 & 01 / 2 \\
19 & 9 \\
16 & 51 / 2 \\
19 & 9\end{array}$ & $\begin{array}{l}4 \text { by } 4 \text { by } 1 / 4 \\
4 \text { by } 4 \text { by } \frac{5}{1} \\
4 \text { by } 4 \text { by } 3 / 8 \\
4 \text { by } 4 \text { by } 3 / 8\end{array}$ & $\begin{array}{l}\frac{11}{16} \\
\frac{11}{16} \\
\frac{11}{16} \\
\frac{11}{16} \\
\frac{11}{16}\end{array}$ & & $\begin{array}{r} \\
6,580 \\
\cdots\end{array}$ & $\begin{array}{r}4,280 \\
4,980\end{array}$ & 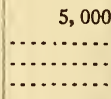 \\
\hline $\begin{array}{l}\mathbf{E} 7 \ldots \ldots \ldots \\
\mathbf{E} 8 \ldots \ldots \ldots\end{array}$ & $\begin{array}{ll}23 & 31 / 2 \\
19 & 9\end{array}$ & $\begin{array}{ll}23 & 01 / 2 \\
19 & 6\end{array}$ & $\begin{array}{l}4 \text { by } 4 \text { by } 3 / 8 \\
4 \text { by } 4 \text { by } 1 / 2\end{array}$ & $\begin{array}{l}\frac{11}{16} \\
\frac{11}{16}\end{array}$ & & & 3,930 & $\begin{array}{r}4,270 \\
\ldots \ldots . \\
\end{array}$ \\
\hline Average.. & & & & & 12,400 & 8,720 & 5,060 & 4,630 \\
\hline
\end{tabular}


TABLE 7.-Results of Compression Tests on Angles with Two or More Bolts in Each Leg.

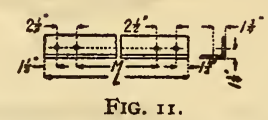

Specimens No. DrI-D2r.

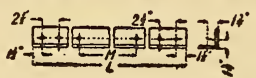

FIG. 12.

Specimens No. $\mathrm{D}_{22}-\mathrm{D}_{37}$

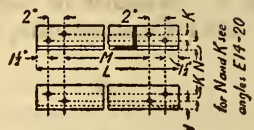

Fig. I3.

Specimens No. Ero-EI2

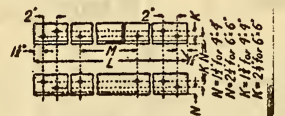

Fig. 14.

Specimens No. $\mathrm{E}_{14}-\mathrm{E}_{20}$.

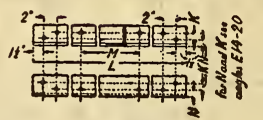

Fig. 15.

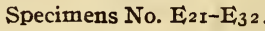

\begin{tabular}{|c|c|c|c|c|c|}
\hline Specimen No. & $\begin{array}{c}\text { Length } \\
\text { L. }\end{array}$ & $\begin{array}{c}\text { Distance } \\
\text { M. }\end{array}$ & angle. & $\begin{array}{c}\text { Distance } \\
\text { N. }\end{array}$ & $\begin{array}{c}\text { Distance } \\
\mathbf{K} .\end{array}$ \\
\hline 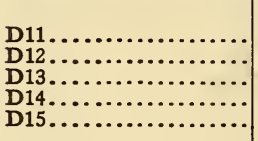 & $\begin{array}{rl}\text { Ft. in. } & \\
5 & 51 / 2 \\
7 & 11 \frac{1}{2} \\
5 & 41 / 2 \\
7 & 10 \\
7 & 10\end{array}$ & $\begin{array}{rl}\text { Ft. } & \text { in. } \\
4 & 91 / 2 \\
7 & 31 / 2 \\
4 & 81 / 2 \\
7 & 2 \\
7 & 2\end{array}$ & 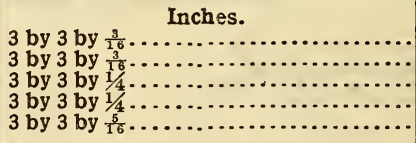 & \begin{tabular}{r|} 
Inches. \\
$13 / 4$ \\
$13 / 4$ \\
$13 / 4$ \\
$13 / 4$ \\
$13 / 4$
\end{tabular} & \begin{tabular}{|l} 
Inches. \\
$\cdots \ldots \ldots$ \\
\hdashline$\ldots \ldots$ \\
$\cdots \cdots$ \\
$\cdots \cdots$
\end{tabular} \\
\hline 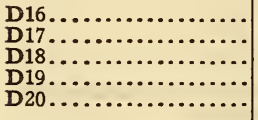 & $\begin{array}{rl}10 & 11 / 2 \\
9 & 1 \\
9 & 1 \\
11 & 111 / 2 \\
11 & 111 / 2\end{array}$ & $\begin{array}{rl}9 & 51 / 2 \\
8 & 5 \\
8 & 5 \\
11 & 31 / 2 \\
11 & 31 / 2\end{array}$ & 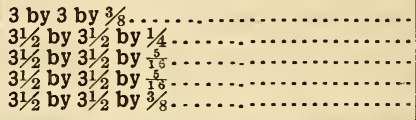 & $\begin{array}{l}13 / 4 \\
13 / 4 \\
13 / 4 \\
13 / 4 \\
13 / 4\end{array}$ & 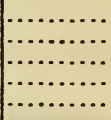 \\
\hline 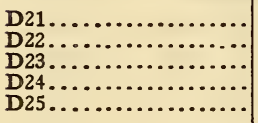 & $\begin{array}{rl}11 & 91 / 2 \\
3 & 2 \\
3 & 11 / 2 \\
3 & 4 \\
5 & 7\end{array}$ & $\begin{array}{rr}11 & 11 / 2 \\
2 & 1 \\
2 & 01 / 2 \\
1 & 10 \\
4 & 6\end{array}$ & 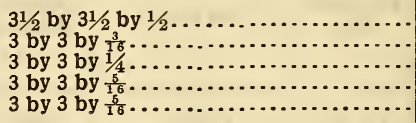 & $\begin{array}{l}13 / 4 \\
13 / 4 \\
13 / 4 \\
1334 \\
13 / 4\end{array}$ & 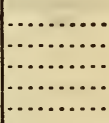 \\
\hline 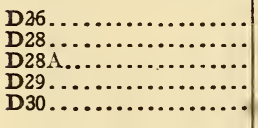 & $\begin{array}{ll}3 & 6 \\
6 & 4 \\
6 & 4 \\
3 & 9 \\
6 & 5\end{array}$ & $\begin{array}{ll}1 & 7 \\
4 & 5 \\
4 & 5 \\
2 & 3 \\
5 & 4\end{array}$ & 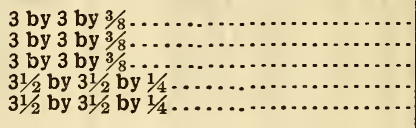 & $\begin{array}{l}13 / 4 \\
13 / 4 \\
13 / 4 \\
13 / 4 \\
13 / 4\end{array}$ & (n..... \\
\hline 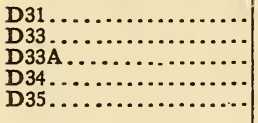 & $\begin{array}{ll}3 & 111 / 2 \\
7 & 8 \\
7 & 8 \\
4 & 2 \\
6 & 71 / 2\end{array}$ & $\begin{array}{lc}2 & 01 / 2 \\
5 & 4 \\
5 & 4 \\
1 & 10 \\
5 & 11 / 2\end{array}$ & 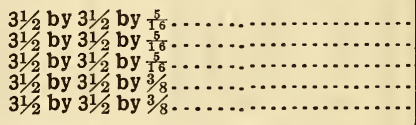 & $\begin{array}{l}13 / 4 \\
13 / 4 \\
13 / 4 \\
13 / 4 \\
13 / 4\end{array}$ & $\begin{array}{l}\cdots \\
\cdots \\
\cdots \cdots \\
\cdots \\
\cdots\end{array}$ \\
\hline 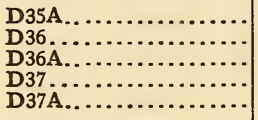 & $\begin{array}{ll}6 & 71 / 2 \\
4 & 4 \\
4 & 4 \\
6 & 9 \\
6 & 9\end{array}$ & $\begin{array}{lr}5 & 11 / 2 \\
1 & 7 \\
1 & 7 \\
4 & 10 \\
4 & 10\end{array}$ & 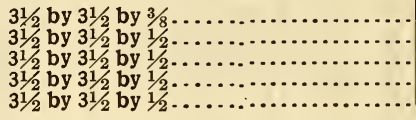 & $\begin{array}{l}13 / 4 \\
13 / 4 \\
13 / 4 \\
13 / 4 \\
13 / 4\end{array}$ & 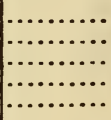 \\
\hline 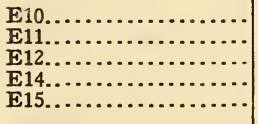 & $\begin{array}{rl}13 & 7 \\
13 & 7 \\
13 & 7 \\
7 & 2 \\
4 & 21 / 2\end{array}$ & $\begin{array}{rl}13 & 0 \\
13 & 0 \\
13 & 0 \\
6 & 3 \\
2 & 71 / 2\end{array}$ & 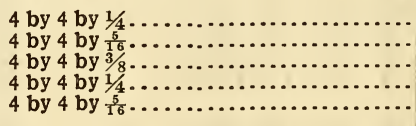 & $\begin{array}{l}11 / 2 \\
11 / 2 \\
11 / 2 \\
11 / 2 \\
11 / 2\end{array}$ & $\begin{array}{l}13 / 8 \\
13 / 8 \\
13 / 8 \\
13 / 8 \\
13 / 8\end{array}$ \\
\hline 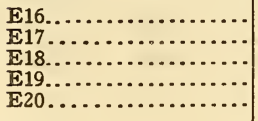 & $\begin{array}{rl}10 & 51 / 2 \\
7 & 5 \\
6 & 21 / 2 \\
15 & 51 / 2 \\
20 & 3\end{array}$ & $\begin{array}{ll}9 & 61 / 2 \\
5 & 10 \\
3 & 111 / 2 \\
14 & 61 / 2 \\
19 & 4\end{array}$ & 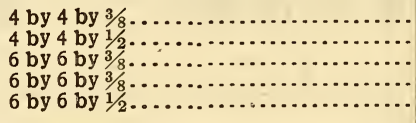 & $\begin{array}{l}11 / 2 \\
11 / 2 \\
21 / 2 \\
21 / 2 \\
21 / 2\end{array}$ & $\begin{array}{l}13 / 8 \\
13 / 8 \\
21 / 4 \\
21 / 4 \\
21 / 4\end{array}$ \\
\hline $\begin{array}{l}\mathrm{E} 21 \ldots \ldots \ldots \\
\mathrm{E} 22 \ldots \ldots \ldots \\
\mathrm{E} 23 \ldots \ldots \ldots \\
\mathrm{E} 24 \ldots \ldots\end{array}$ & $\begin{array}{ll}4 & 01 / 2 \\
7 & 4 \\
4 & 41 / 2 \\
7 & 4 \\
4 & 8\end{array}$ & $\begin{array}{ll}2 & 91 / 2 \\
6 & 1 \\
2 & 51 / 2 \\
6 & 1 \\
2 & 1\end{array}$ & 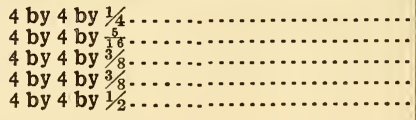 & $\begin{array}{l}11 / 2 \\
11 / 2 \\
11 / 2 \\
11 / 2 \\
11 / 2\end{array}$ & $\begin{array}{l}13 / 8 \\
13 / 8 \\
13 / 8 \\
13 / 8 \\
13 / 8\end{array}$ \\
\hline 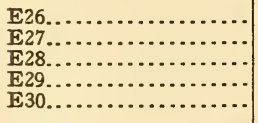 & $\begin{array}{rc}10 & 8 \\
6 & 4 \\
10 & 11 \\
15 & 6 \\
6 & 111 / 2\end{array}$ & $\begin{array}{rl}9 & 5 \\
3 & 9 \\
9 & 0 \\
14 & 3 \\
3 & 01 / 2\end{array}$ & $\begin{array}{l}6 \text { by } 6 \text { by } 3 / 8 . \\
6 \text { by } 6 \text { by } 1 / 2 . \\
6 \text { by } 6 \text { by } 1 / 2 . \\
6 \text { by } 6 \text { by } 1 / 2 \\
6 \text { by } 6 \text { by } 3 / 4 .\end{array}$ & $\begin{array}{l}21 / 2 \\
21 / 2 \\
21 / 2 \\
21 / 2 \\
21 / 2\end{array}$ & $\begin{array}{l}21 / 4 \\
21 / 4 \\
21 / 4 \\
21 / 4 \\
21 / 4\end{array}$ \\
\hline $\begin{array}{l}\text { E30A.. } \\
\text { E31... } \\
\text { E32.... }\end{array}$ & $\begin{array}{ll}6 & 111 / 2 \\
11 & 2 \\
12 & 6\end{array}$ & $\begin{array}{ll}3 & 01 / 2 \\
8 & 7 \\
8 & 7\end{array}$ & $\begin{array}{l}6 \text { by } 6 \text { by } 3 / 4 . \\
6 \text { by } 6 \text { by } 3 / 4 . \\
6 \text { by } 6 \text { by } 3 / 4 .\end{array}$ & $\begin{array}{l}21 / 2 \\
21 / 2 \\
21 / 2\end{array}$ & $\begin{array}{l}21 / 4 \\
21 / 4 \\
21 / 4\end{array}$ \\
\hline
\end{tabular}


TABLE 7.-Results of Compression Tests on Angles with Two or More Bolts in Each Leg-Continued.

\begin{tabular}{|c|c|c|c|c|c|c|}
\hline \multirow{2}{*}{ Specimen No. } & \multirow{2}{*}{$\begin{array}{l}\text { Diam- } \\
\text { eter of } \\
\text { holes. }\end{array}$} & \multirow{2}{*}{$\begin{array}{c}\text { Number } \\
\text { of holes } \\
\text { in each } \\
\text { leg. }\end{array}$} & \multicolumn{4}{|c|}{$\begin{array}{l}\left.\text { Maximum load (lbs. } / \text { in. }{ }^{2}\right) \\
\text { ness ratio } 1 / \mathrm{r}=\end{array}$} \\
\hline & & & 50 & 100 & $150^{-}$ & 200 \\
\hline 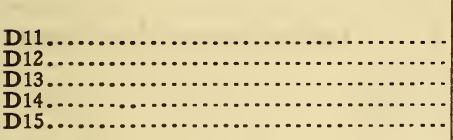 & 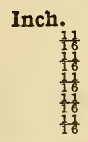 & $\begin{array}{l}2 \\
2 \\
2 \\
2 \\
2\end{array}$ & 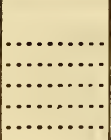 & $\begin{array}{r}36,800 \\
32,400 \\
\cdots \\
\cdots\end{array}$ & $\begin{array}{r}26,050 \\
29,900 \\
29,900\end{array}$ & 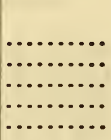 \\
\hline 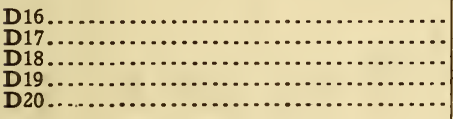 & $\begin{array}{l}\frac{11}{16} \\
\frac{1}{116} \\
\frac{1}{16} \\
\frac{11}{71} \\
\frac{11}{16} \\
\frac{1}{16}\end{array}$ & $\begin{array}{l}2 \\
2 \\
2 \\
2 \\
2\end{array}$ & 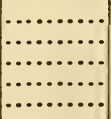 & $\mid$\begin{tabular}{ll|}
$\cdots \cdots \cdots$ \\
$\cdots \cdots \cdots$ \\
$\cdots \cdots \cdots$ \\
$\cdots \cdots \cdots$ \\
$\cdots \cdots \cdots$
\end{tabular} & $\begin{array}{r}25,000 \\
25,000 \\
\cdots \\
\cdots\end{array}$ & $\begin{array}{r}22,700 \\
\cdots . \\
17,700 \\
19,900\end{array}$ \\
\hline 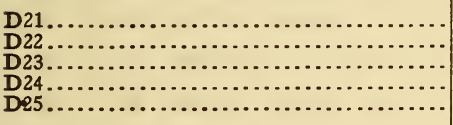 & $\begin{array}{l}\frac{11}{16} \\
\frac{11}{16} \\
\frac{11}{16} \\
\frac{11}{16} \\
\frac{1}{16} \\
\frac{1}{16}\end{array}$ & $\begin{array}{l}2 \\
3 \\
3 \\
4 \\
3\end{array}$ & $\begin{array}{r}41,000 \\
35,500 \\
36,000 \\
\ldots \ldots \ldots \ldots\end{array}$ & \begin{tabular}{r}
$\cdots$ \\
$\cdots$ \\
\hdashline$\ldots \ldots \ldots$ \\
35,300
\end{tabular} & 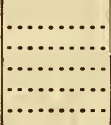 & $\begin{array}{l}15,800 \\
\ldots \ldots \\
\cdots \ldots \\
\cdots \ldots\end{array}$ \\
\hline $\begin{array}{l}\text { D } 26 \ldots \ldots \\
\text { D } 28 \\
\text { D } 28 \mathbf{A} \\
\text { D } 29 \ldots \ldots \\
\text { D } 30 \ldots \ldots\end{array}$ & $\begin{array}{l}\frac{11}{116} \\
\frac{11}{1 \frac{1}{16}} \\
\frac{1}{16} \\
\frac{11}{11} \\
\frac{1}{16} \\
\frac{2}{16}\end{array}$ & $\begin{array}{l}5 \\
5 \\
5 \\
4 \\
3\end{array}$ & $\begin{array}{r}35,500 \\
\cdots \\
35,000 \\
\cdots\end{array}$ & $\begin{array}{r}33,800 \\
31,600 \\
33,000\end{array}$ & $\mid \begin{array}{ll} \\
\cdots \cdots \\
\cdots \cdots\end{array}$ & ( \\
\hline 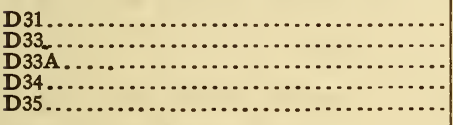 & $\begin{array}{l}\frac{11}{16} \\
\frac{11}{16} \\
\frac{11}{16} \\
\frac{11}{16} \\
\frac{11}{16}\end{array}$ & $\begin{array}{l}5 \\
6 \\
6 \\
6 \\
4\end{array}$ & $\begin{array}{r}36,000 \\
38,000 \\
3 . \\
\cdots\end{array}$ & $\begin{array}{r}34,000 \\
35,700 \\
34,900\end{array}$ & $\begin{array}{l}\cdots \cdots \\
\cdots \cdots \\
\cdots \cdots \\
\cdots \cdots \\
\cdots \cdots\end{array}$ & $\begin{array}{ll}\cdots \\
\cdots \cdots\end{array}$ \\
\hline 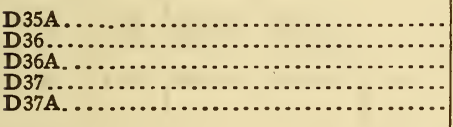 & $\begin{array}{l}\frac{11}{16} \\
\frac{11}{16} \\
\frac{1}{16} \\
\frac{11}{16} \\
\frac{11}{16}\end{array}$ & $\begin{array}{l}4 \\
7 \\
7 \\
5 \\
5\end{array}$ & $\begin{array}{r}41,800 \\
41,200 \\
\cdots \\
\cdots\end{array}$ & \begin{tabular}{r}
34,000 \\
\hdashline 3. \\
34,100 \\
33,900
\end{tabular} & 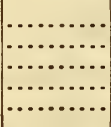 & $\begin{array}{ll}\cdots \cdots \\
\cdots \cdots \\
\cdots \cdots \\
\cdots \cdots\end{array}$ \\
\hline $\begin{array}{l}\text { E10 } \\
\text { E11 } 11 \ldots \\
\text { E12 } \\
\text { E14 } 14 \ldots \\
\text { E15 }\end{array}$ & $\begin{array}{l}\frac{11}{16} \\
\frac{11}{16} \\
\frac{1}{16} \\
\frac{1}{16} \\
\frac{11}{16} \\
\frac{11}{16}\end{array}$ & $\begin{array}{l}2 \\
2 \\
2 \\
3 \\
5\end{array}$ & 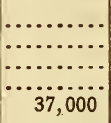 & $\mid \begin{array}{c}\cdots \\
33,500 \\
\cdots \cdots\end{array}$ & & $\begin{array}{r}14,250 \\
16,700 \\
20,150 \\
\ldots . . . \\
\ldots . . .\end{array}$ \\
\hline 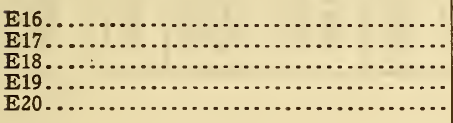 & $\begin{array}{l}\frac{11}{16} \\
\frac{11}{11} \\
\frac{13}{16} \\
\frac{13}{16} \\
\frac{13}{16} \\
\frac{23}{16}\end{array}$ & $\begin{array}{l}3 \\
5 \\
7 \\
3 \\
3\end{array}$ & $\begin{array}{r}31,600 \\
\cdots \ldots \ldots \\
\cdots\end{array}$ & 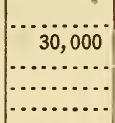 & $\begin{array}{r}25,600 \\
22,800 \\
\cdots\end{array}$ & $\begin{array}{r}\cdots \\
20,000\end{array}$ \\
\hline 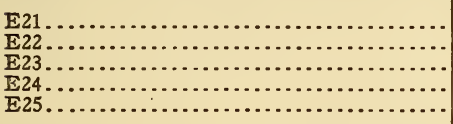 & $\begin{array}{l}\frac{11}{16} \\
\frac{11}{16} \\
\frac{11}{21} \\
\frac{11}{16} \\
\frac{11}{16} \\
\frac{11}{16}\end{array}$ & $\begin{array}{l}4 \\
4 \\
6 \\
4 \\
8\end{array}$ & $\begin{array}{r}37,500 \\
36,200 \\
31,000\end{array}$ & $\begin{array}{r}34,480 \\
33,000 \\
\cdots\end{array}$ & $\begin{array}{l}\cdots \ldots . \\
\cdots \ldots . \\
\cdots \ldots . \\
\cdots \ldots . \\
\ldots \ldots\end{array}$ & 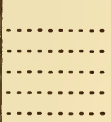 \\
\hline 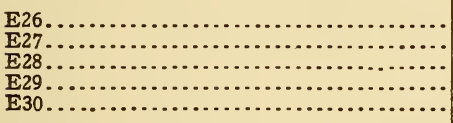 & $\begin{array}{l}\frac{13}{16} \\
\frac{18}{16} \\
\frac{19}{16} \\
\frac{13}{16} \\
\frac{13}{16} \\
\frac{3}{16}\end{array}$ & $\begin{array}{r}4 \\
8 \\
6 \\
4 \\
12\end{array}$ & $\begin{array}{r}28,300 \\
\cdots \\
\hdashline 31,700\end{array}$ & $\begin{array}{r}30,200 \\
27,900 \\
\cdots \\
\cdots\end{array}$ & $\begin{array}{r} \\
27,750 \\
\cdots \ldots .\end{array}$ & 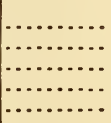 \\
\hline $\begin{array}{l}\text { E30A } \\
\text { E31 } \\
\text { E32 }\end{array}$ & $\begin{array}{l}\frac{13}{16} \\
\frac{13}{166} \\
\frac{13}{16}\end{array}$ & $\begin{array}{r}12 \\
8 \\
12\end{array}$ & $\begin{array}{r}32,150 \\
\cdots \\
\cdots\end{array}$ & $\begin{array}{r}35,000 \\
28,820\end{array}$ & $\cdots$ & $\cdots$ \\
\hline
\end{tabular}

$109714^{\circ}-22-2$ 
TABLE 8.- Results of Tests on Coupon Specimens.

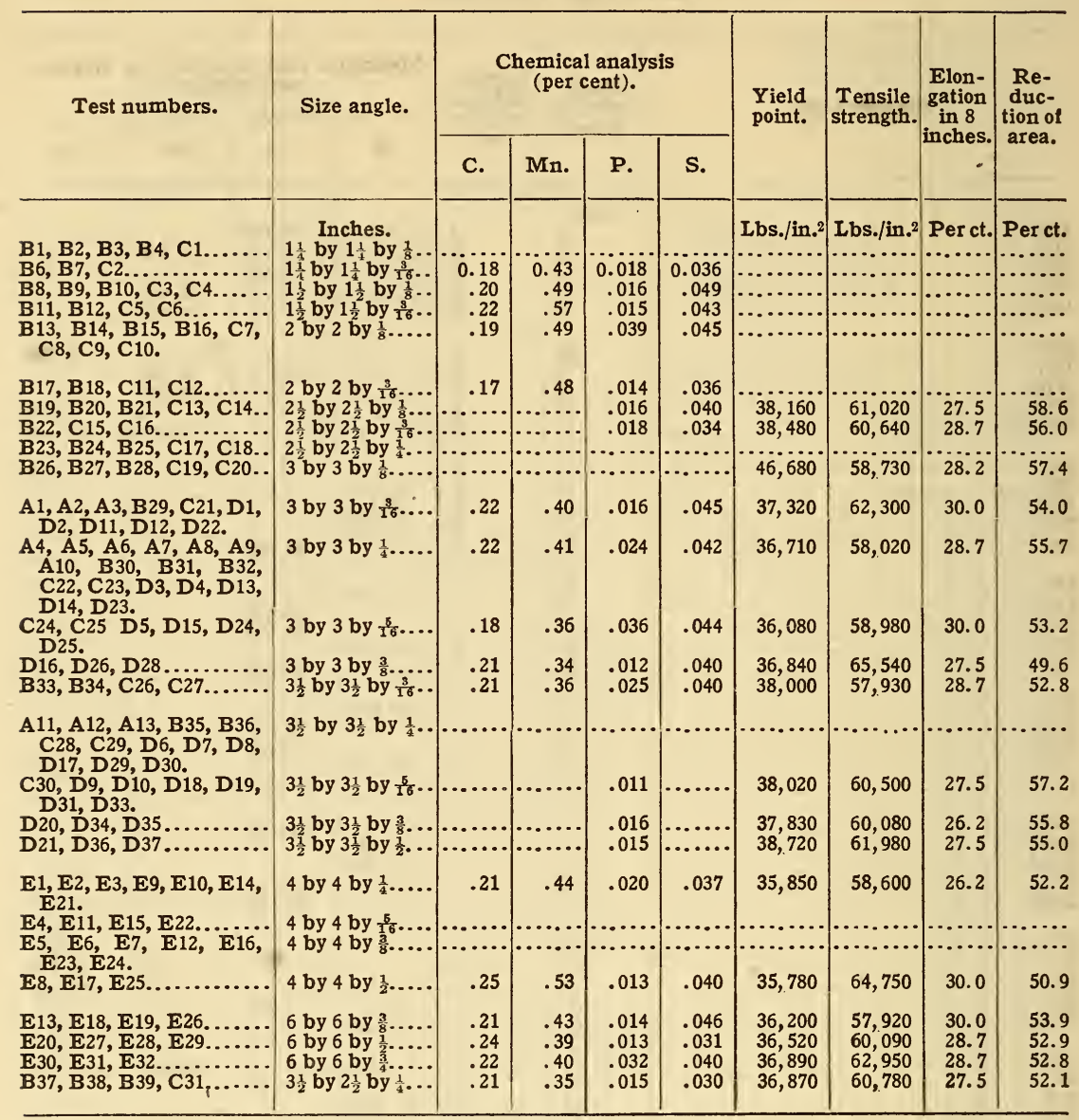


TABLE 9.-Comparison of Lateral Deflection to Strength of Angles with One Bolt Connection.

\begin{tabular}{|c|c|c|c|c|c|}
\hline \multirow{2}{*}{ Specimen No. } & \multirow{2}{*}{$\begin{array}{c}\text { Slender- } \\
\text { ness } \\
\text { ratio. }\end{array}$} & \multirow{2}{*}{$\begin{array}{l}\text { Lateral } \\
\text { deflection } \\
\text { at } 4 / 9 \mathrm{~S} \text {. }\end{array}$} & \multicolumn{3}{|c|}{ Rank. } \\
\hline & & & $\begin{array}{c}\text { By } \\
\text { strength. }\end{array}$ & $\begin{array}{c}\text { By deflec- } \\
\text { tion. }\end{array}$ & $\begin{array}{l}\text { Differ- } \\
\text { ence in- }\end{array}$ \\
\hline 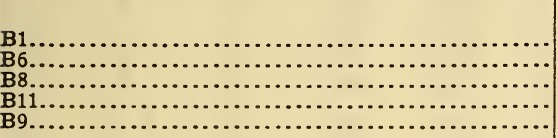 & $\begin{array}{r}1 / \mathbf{r} \\
200 \\
200 \\
200 \\
200 \\
250\end{array}$ & \begin{tabular}{r|} 
Inch. \\
0.11 \\
.06 \\
.17 \\
.16 \\
.10
\end{tabular} & $\begin{array}{l}3 \\
1 \\
4 \\
2 \\
1\end{array}$ & $\begin{array}{l}2 \\
1 \\
4 \\
3 \\
1\end{array}$ & $\begin{array}{l}1 \\
0 \\
0 \\
1 \\
0\end{array}$ \\
\hline $\begin{array}{l}\text { B13 } \\
\mathbf{B} 17 \\
\mathbf{B} 19 \\
\mathbf{B} 23 \\
\mathbf{B} 26 \ldots\end{array}$ & $\begin{array}{l}250 \\
250 \\
250 \\
250 \\
250\end{array}$ & $\begin{array}{l}.20 \\
.23 \\
.19 \\
.19 \\
.33\end{array}$ & $\begin{array}{r}8 \\
3-5 \\
3-5 \\
2 \\
10\end{array}$ & $\begin{array}{r}4 \\
5 \\
2-3 \\
2-3 \\
10\end{array}$ & $\begin{array}{l}4 \\
0 \\
0 \\
0 \\
0\end{array}$ \\
\hline 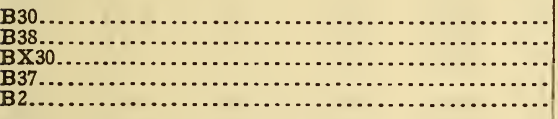 & $\begin{array}{r}250 \\
250 \\
250 \\
250 \\
300\end{array}$ & $\begin{array}{l}.25 \\
.31 \\
.25 \\
.32 \\
.13\end{array}$ & \begin{tabular}{r|}
$3-5$ \\
7 \\
6 \\
9 \\
2
\end{tabular} & $\begin{array}{r}6-7 \\
8 \\
6-7 \\
9 \\
1\end{array}$ & $\begin{array}{l}1 \\
1 \\
0 \\
0 \\
1\end{array}$ \\
\hline 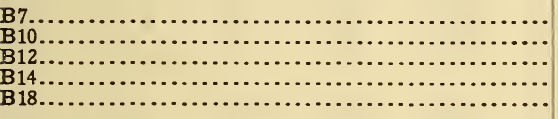 & $\begin{array}{l}300 \\
300 \\
300 \\
300 \\
300\end{array}$ & $\begin{array}{l}.15 \\
.16 \\
.15 \\
.17 \\
.24\end{array}$ & $\begin{array}{r}3 \\
5 \\
1 \\
8-10 \\
6-7\end{array}$ & $\begin{array}{r}2-3 \\
4 \\
2-3 \\
5 \\
10-11\end{array}$ & $\begin{array}{l}0 \\
1 \\
1 \\
3 \\
3\end{array}$ \\
\hline 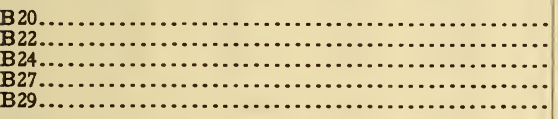 & $\begin{array}{l}300 \\
300 \\
300 \\
300 \\
300\end{array}$ & $\begin{array}{l}.54 \\
.25 \\
.20 \\
.73 \\
.21\end{array}$ & $\begin{array}{r}16 \\
8-10 \\
4 \\
17 \\
12\end{array}$ & $\begin{array}{r}16 \\
12 \\
6-7 \\
17 \\
8\end{array}$ & $\begin{array}{l}0 \\
2 \\
2 \\
0 \\
4\end{array}$ \\
\hline 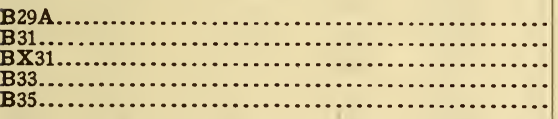 & $\begin{array}{l}300 \\
300 \\
300 \\
300 \\
300\end{array}$ & $\begin{array}{l}.24 \\
.32 \\
.23 \\
.20 \\
.40\end{array}$ & $\begin{array}{r}13 \\
11 \\
6-7 \\
8-10 \\
14\end{array}$ & $\begin{array}{r}10-11 \\
13 \\
9 \\
6-7 \\
14\end{array}$ & $\begin{array}{l}2 \\
2 \\
2 \\
1 \\
0\end{array}$ \\
\hline 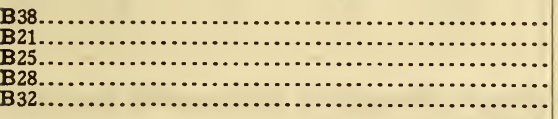 & $\begin{array}{l}300 \\
350 \\
350 \\
350 \\
350\end{array}$ & $\begin{array}{l}.49 \\
.36 \\
.27 \\
.42 \\
.64\end{array}$ & \begin{tabular}{r|}
15 \\
7 \\
2 \\
8 \\
5
\end{tabular} & $\begin{array}{r}15 \\
5 \\
4 \\
6 \\
8\end{array}$ & $\begin{array}{l}0 \\
2 \\
2 \\
2 \\
3\end{array}$ \\
\hline 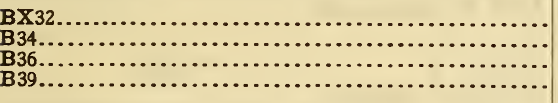 & $\begin{array}{l}350 \\
350 \\
350 \\
350\end{array}$ & $\begin{array}{l}.15 \\
.21 \\
.50 \\
.20\end{array}$ & $\begin{array}{l}3 \\
4 \\
6 \\
1\end{array}$ & $\begin{array}{l}1 \\
3 \\
7 \\
2\end{array}$ & $\begin{array}{l}2 \\
1 \\
1 \\
1\end{array}$ \\
\hline
\end{tabular}


TABLE 10.-Comparison of Lateral Deflection to Strength of Angles with Two Bolt Connections in One Leg Only.

\begin{tabular}{|c|c|c|c|c|c|}
\hline \multirow{2}{*}{ Specimen No. } & \multirow{2}{*}{$\begin{array}{l}\text { Slender- } \\
\text { ness } \\
\text { ratio. }\end{array}$} & \multirow{2}{*}{$\begin{array}{c}\text { Lateral } \\
\text { deflection } \\
\text { at } 4 / 9 \mathrm{~S} .\end{array}$} & \multicolumn{3}{|c|}{ Rank. } \\
\hline & & & $\underset{\text { strength. }}{\text { By }}$ & $\begin{array}{c}\text { By deflec- } \\
\text { tion. }\end{array}$ & $\begin{array}{l}\text { Differ- } \\
\text { ence in- }\end{array}$ \\
\hline $\begin{array}{l}\mathrm{C} 4 \\
\mathrm{C} 6 \ldots \\
\mathrm{C} 7 \ldots \ldots \\
\mathrm{C} 11, \ldots \ldots\end{array}$ & $\begin{array}{r}1 / \mathbf{r} \\
150 \\
150 \\
150 \\
150 \\
150\end{array}$ & $\begin{array}{r}\text { Inch. } \\
0.12 \\
.10 \\
.23 \\
.17 \\
.32\end{array}$ & $\begin{array}{l}2 \\
1 \\
7 \\
3 \\
9\end{array}$ & \begin{tabular}{l|l}
2 \\
1 \\
5 \\
3 \\
9
\end{tabular} & $\begin{array}{l}0 \\
0 \\
2 \\
0 \\
0\end{array}$ \\
\hline $\begin{array}{l}\mathrm{C} 15 \\
\mathrm{C} 17 \ldots \ldots \\
\mathrm{C} 19 \ldots \ldots \\
\mathrm{C} 22, \ldots \ldots\end{array}$ & $\begin{array}{l}150 \\
150 \\
150 \\
150 \\
150\end{array}$ & $\begin{array}{l}.25 \\
.20 \\
.23 \\
.27 \\
.25\end{array}$ & $\begin{array}{r}4 \\
5 \\
10 \\
8 \\
6\end{array}$ & $\begin{array}{r}6-7 \\
4 \\
10 \\
8 \\
6-7\end{array}$ & $\begin{array}{l}2 \\
1 \\
0 \\
0 \\
0\end{array}$ \\
\hline 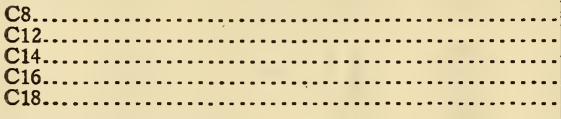 & $\begin{array}{l}200 \\
200 \\
200 \\
200 \\
200\end{array}$ & $\begin{array}{l}.19 \\
.13 \\
.24 \\
.20 \\
.29\end{array}$ & $\begin{array}{r}1 \\
2 \\
13 \\
3 \\
5\end{array}$ & \begin{tabular}{r|r|r}
$2-3$ \\
1 \\
$6-9$ \\
4 \\
14
\end{tabular} & $\begin{array}{l}1 \\
1 \\
4 \\
1 \\
9\end{array}$ \\
\hline 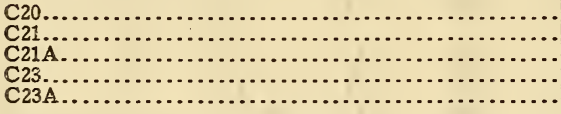 & $\begin{array}{l}200 \\
200 \\
200 \\
200 \\
200\end{array}$ & \begin{tabular}{l|}
.26 \\
.24 \\
.24 \\
.26 \\
.24
\end{tabular} & $\begin{array}{r}16 \\
8 \\
4 \\
11 \\
7\end{array}$ & \begin{tabular}{r|}
12 \\
$6-9$ \\
$6-9$ \\
11 \\
$6-9$
\end{tabular} & $\begin{array}{l}4 \\
0 \\
2 \\
0 \\
0\end{array}$ \\
\hline $\begin{array}{l}\mathrm{C} 24 \ldots \\
\mathrm{C} 24 \mathrm{~A} \\
\mathrm{C} 26 \ldots \ldots \\
\mathrm{C} 28 \ldots\end{array}$ & $\begin{array}{l}200 \\
200 \\
200 \\
200 \\
200\end{array}$ & $\begin{array}{l}.27 \\
.23 \\
.25 \\
.35 \\
.30\end{array}$ & $\begin{array}{r}9-10 \\
6 \\
12 \\
15 \\
14\end{array}$ & $\begin{array}{r}13 \\
5 \\
10 \\
16 \\
15\end{array}$ & $\begin{array}{l}3 \\
1 \\
2 \\
1 \\
1\end{array}$ \\
\hline 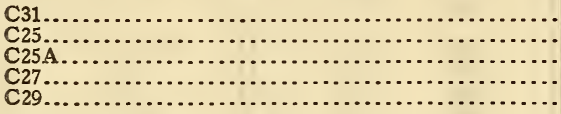 & $\begin{array}{l}200 \\
250 \\
250 \\
250 \\
250\end{array}$ & $\begin{array}{l}.19 \\
.27 \\
\cdots \\
.30 \\
.64\end{array}$ & $\begin{array}{r}9-10 \\
1 \\
4-5 \\
4-5 \\
7\end{array}$ & \begin{tabular}{r|}
$2-3$ \\
1 \\
4 \\
2 \\
7
\end{tabular} & $\begin{array}{l}6 \\
0 \\
2 \\
0\end{array}$ \\
\hline $\begin{array}{l}\mathrm{C} 29 \mathrm{~A} \\
\mathrm{C} 30 \\
\mathrm{C} 30 \mathrm{~A}\end{array}$ & $\begin{array}{l}250 \\
250 \\
250\end{array}$ & $\begin{array}{l}.32 \\
.35 \\
.31\end{array}$ & $\begin{array}{l}6 \\
3 \\
2\end{array}$ & $\begin{array}{l}5 \\
6 \\
3\end{array}$ & \\
\hline
\end{tabular}

TABLE 11.-Comparison of Lateral Deflection to Strength of Angles with Two Bolt Connections, One Bolt in Each Leg.

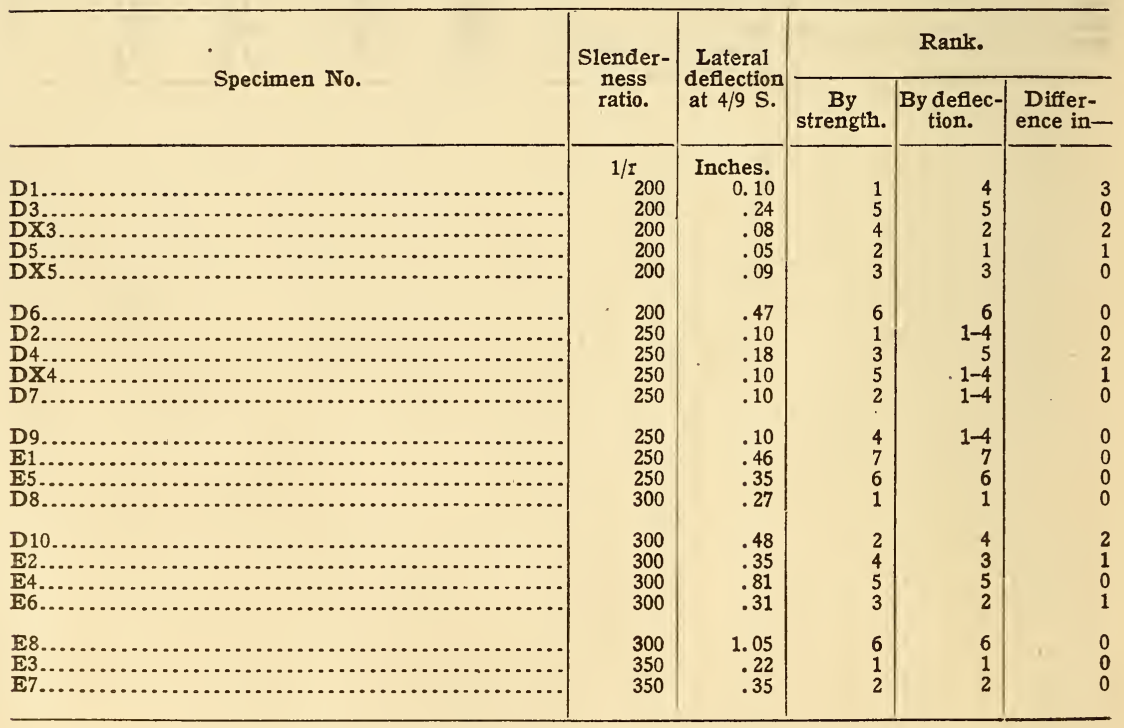


TABLE 12.-Comparison of Lateral Deflection to Strength of Angles with Two or More Bolts in Each Leg.

\begin{tabular}{|c|c|c|c|c|c|}
\hline 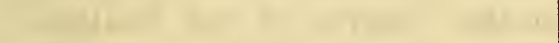 & Slender- & Lat & \multicolumn{3}{|c|}{ Rank. } \\
\hline Specimen 1 o. & ratio. & at $4 / 9 \mathrm{~S}$. & $\begin{array}{l}\text { By } \\
\text { strength. }\end{array}$ & $\begin{array}{c}\text { By deflec- } \\
\text { tion. }\end{array}$ & $\begin{array}{l}\text { Differ- } \\
\text { ence in- }\end{array}$ \\
\hline 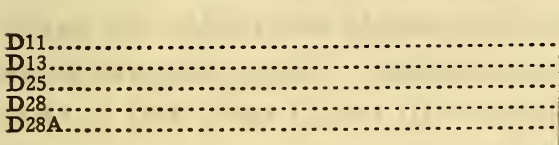 & $\begin{array}{r}1 / \mathbf{r} \\
100 \\
100 \\
100 \\
100 \\
100\end{array}$ & $\begin{array}{r}\text { Inch. } \\
0.03 \\
.05 \\
.05 \\
.09 \\
.05\end{array}$ & $\begin{array}{r}1 \\
15 \\
3 \\
11 \\
16\end{array}$ & $\begin{array}{r}2 \\
5-14 \\
5-14 \\
17 \\
5-14\end{array}$ & \\
\hline 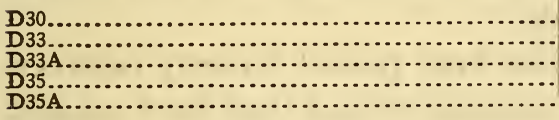 & $\begin{array}{l}100 \\
100 \\
100 \\
100 \\
100\end{array}$ & $\begin{array}{l}.05 \\
.05 \\
.04 \\
.05 \\
.05\end{array}$ & $\begin{array}{r}13 \\
8 \\
2 \\
5 \\
9\end{array}$ & $\begin{array}{r}5-14 \\
5-14 \\
3 \\
5-14 \\
5-14\end{array}$ & $\begin{array}{l}0 \\
0 \\
1\end{array}$ \\
\hline 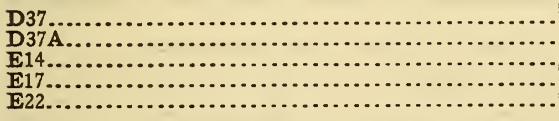 & $\begin{array}{l}100 \\
100 \\
100 \\
100 \\
100\end{array}$ & $\begin{array}{l}.05 \\
.05 \\
.05 \\
.08 \\
.02\end{array}$ & $\begin{array}{r}7 \\
10 \\
12 \\
18 \\
6\end{array}$ & $\begin{array}{r}5-14 \\
5-14 \\
5-14 \\
16 \\
1\end{array}$ & $\begin{array}{l}0 \\
0 \\
0 \\
2 \\
5\end{array}$ \\
\hline $\begin{array}{l}\mathbf{E} 24 \\
\mathbf{E} 26 \\
\mathbf{E} 28 \\
\mathbf{E} 31 \\
\mathbf{E} 32 \ldots\end{array}$ & $\begin{array}{l}100 \\
100 \\
100 \\
100 \\
100\end{array}$ & $\begin{array}{r}.08 \\
.30 \\
\cdots \\
.12\end{array}$ & $\begin{array}{r}14 \\
17 \\
20 \\
4 \\
19\end{array}$ & $\begin{array}{r}15 \\
19 \\
20 \\
4 \\
18\end{array}$ & 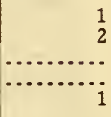 \\
\hline 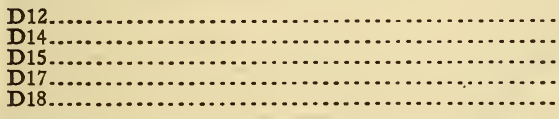 & $\begin{array}{l}150 \\
150 \\
150 \\
150 \\
150\end{array}$ & $\begin{array}{l}.10 \\
.05 \\
.09 \\
.10 \\
.06\end{array}$ & $\begin{array}{r}4 \\
1-2 \\
1-2 \\
6-7 \\
6-7\end{array}$ & $\begin{array}{r}4-5 \\
1 \\
3 \\
4-5 \\
2\end{array}$ & $\begin{array}{l}0 \\
0 \\
1\end{array}$ \\
\hline 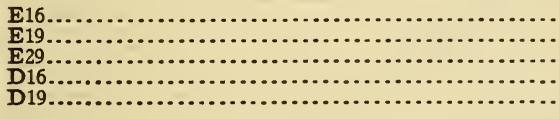 & $\begin{array}{l}150 \\
150 \\
150 \\
200 \\
200\end{array}$ & $\begin{array}{l}.15 \\
.22 \\
.22 \\
.10 \\
.16\end{array}$ & $\begin{array}{l}5 \\
8 \\
3 \\
1 \\
4\end{array}$ & $\begin{array}{r}6 \\
7-8 \\
7-8 \\
4 \\
6\end{array}$ & $\begin{array}{l}1 \\
0 \\
4\end{array}$ \\
\hline 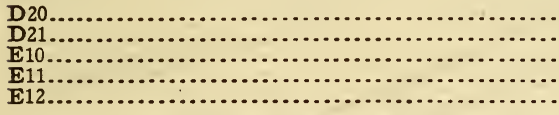 & $\begin{array}{l}200 \\
200 \\
200 \\
200 \\
200\end{array}$ & $\begin{array}{l}.05 \\
.12 \\
.19 \\
.07 \\
.03\end{array}$ & $\begin{array}{l}3 \\
6 \\
7 \\
5 \\
2\end{array}$ & $\begin{array}{l}2 \\
5 \\
7 \\
3 \\
1\end{array}$ & \\
\hline
\end{tabular}

TABLE 13.-End Fixation Factors for Various End Connections of Angles.

\begin{tabular}{|c|c|c|c|}
\hline End connection. & $\begin{array}{l}\text { Fixation } \\
\text { factor. }\end{array}$ & End connection. & $\begin{array}{l}\text { Fixation } \\
\text { factor. }\end{array}$ \\
\hline $\begin{array}{l}\text { Angles with square ends, no bolts ........ } \\
\text { Two or more bolts in each leg............ } \\
\text { Two bolts, in one leg only............... }\end{array}$ & $\begin{array}{l}1.9 \\
1.5 \\
1.3\end{array}$ & $\begin{array}{l}\text { Two bolts, one in each leg } \ldots \ldots \ldots \ldots \ldots \ldots \\
\text { One bolt } \\
\text { Ends folded } . \cdots \cdots \cdots \\
\end{array}$ & $\begin{array}{l}1.3 \\
1.1 \\
1.1\end{array}$ \\
\hline
\end{tabular}

\section{RESULTS AND DISCUSSION OF TESTS.}

(a) General Discussion.-The value of the maximum load sustained by each column was measured. These values are given in Tables $I$ to 7 , inclusive, and have been plotted against the values of the slenderness ratio $l / r$ in Figures $\mathrm{I} 6$ and $\mathrm{I} 7$. In these figures the average value of the maximum loads for each slenderness ratio is shown by a solid circle. Full lines connect these average values. It will be noted from Figure I 7 that for any given slenderness ratio the individual results are quite scattered, and when conclusions are drawn from the average values this fact must be kept in mind. 
The manner in which the angles were held in the testing machine exerted a great influence on their strength. In other words, the strength of a column varies with the "degree of end fixation." The amount of this "end fixation" may be expressed by a fixation factor $f=l / L$, where $l$ is the actual length of the member and $L$ the length of the round end member which would fail under the same load; i. e., the "free length" of the member. Thus, the end fixation factor would be 1.0 for a column with round ends and 2.0 for a specimen tested with fixed ends.

The angles with square ends that were placed directly between the head and base of the testing machine would thus have an end

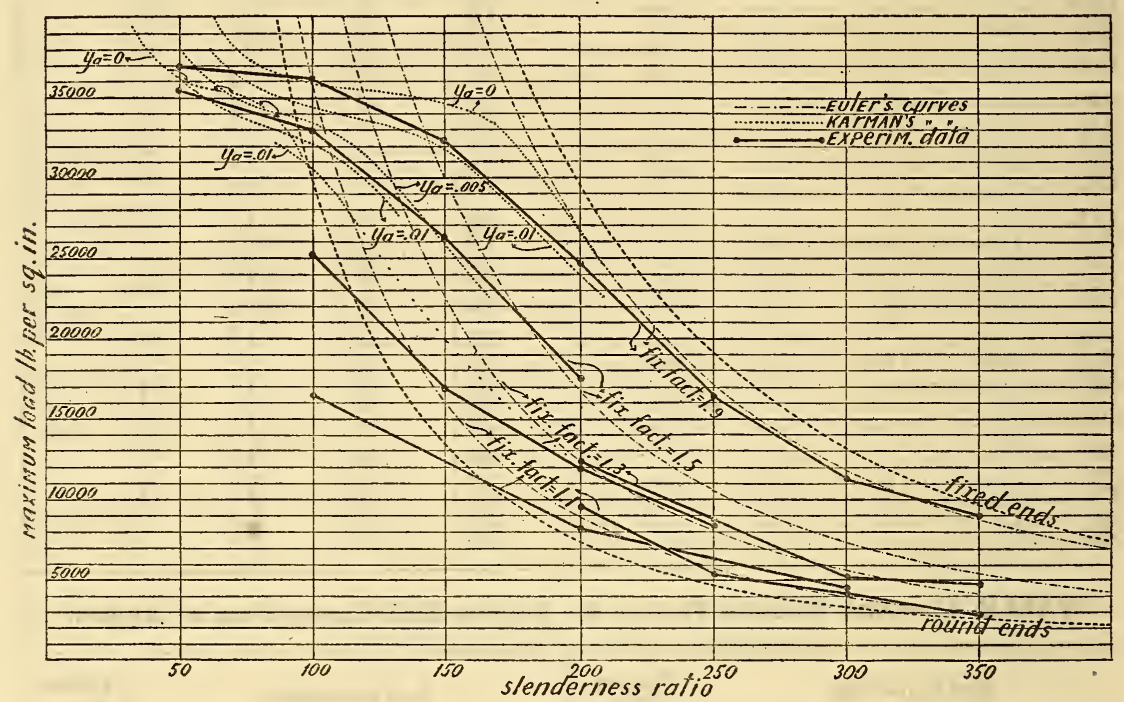

FIG. I6.-Relation of maximum load to slenderness ratio.

fixation of approximately the same degree as a column with theoretically fixed ends under axial loading, while specimens that were held with one bolt in one leg would be expected to approximate a round-end specimen under eccentric loading. When more than one bolt is used, the fixation factor would increase and approach 2.0 as the limit for the most rigidly held columns. End fixation factors for various end connections are given in Table I3.

It must be pointed out that in such column tests there is always present some eccentricity, due to imperfect centering in the testing machine and also to the manner in which the load is applied to the specimen, as by bolted connections. It is very difficult to accurately center even a short compression test specimen. The load was eccentric for all the angles bolted to their end con- 
nections, and this eccentricity of loading always produces a diminution of the maximum load.

The results of the tests were compared with several types of column formulas. Formulas of the Rankine-Gordon type represent the results fairly well for values of the slenderness ratio up to about 150 , but the longer column results are evidently best represented by the Euler formula:

$$
\frac{P}{a}=\frac{\pi^{2} E}{\left(\frac{l}{f r}\right)^{2}}
$$

where $P=$ total load, pounds.

$a=$ cross-section area, square inches.

$E=$ modulus of elasticity, pounds per square inch.

$l=$ length of column, inches.

$r=$ radius of gyration, inches.

and $\quad f=$ fixation factor.

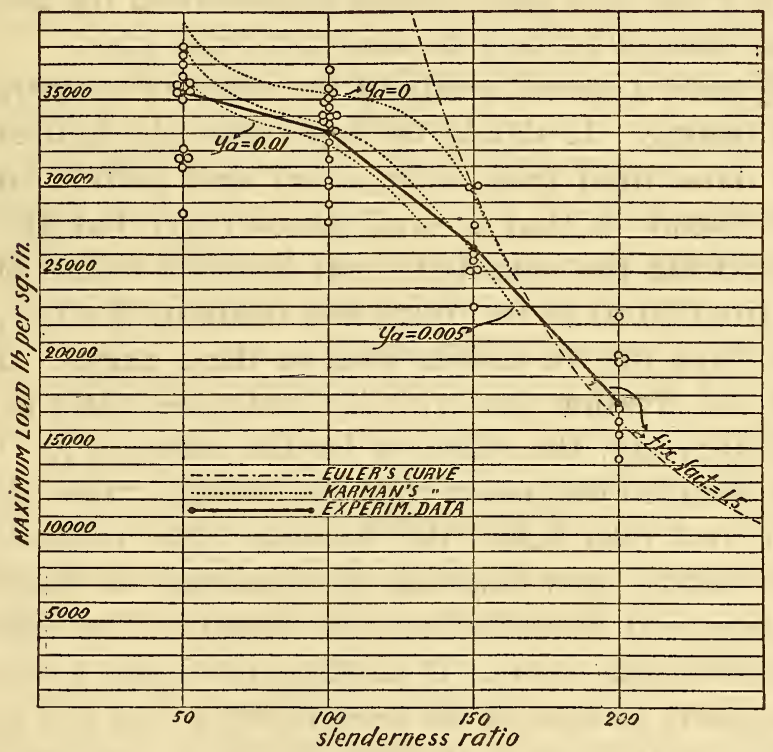

FIG. I7.-Relation of maximum load to slenderness ratio for fixation factor $f=$ I.5.

The Euler formula does not give the strength of short columns, however, since for such lengths the elastic limit of the material is passed before the column fails. The theory of the deviation of short columns from Euler's law has been worked out by Considére (K. Considére, Résistance des Pièces Comprimées, Comptes Rendus, Congrès International des Procédés de Construction, pp. 371-397, I89I), Jasinsky (Jasinsky, Zu den Knickfragen, Schweiz. Bauzeitung, vol. 25, p. I72, I895), and Kármán (Theo. von Kár- 
mán, Untersuchung über Knickfestigkeit, Forschungsarbeiten a. d. Gebiete d. Ingenieurwesens, No. 8I, I910), and later independently by Southwell (R. V. Southwell, The Strength of Struts, Engineering, vol. 94, pp. 248-250, I9I2; Aircraft Engineering, vol. I, p. 20 et seq., January, 1920). The theoretical curve given by Kármán for a steel whose physical properties had been determined were recalculated for a yield point of $37,000 \mathrm{lbs}$./in. ${ }^{2}$ and a modulus of elasticity of $30,000,000 \mathrm{lbs}$./in. ${ }^{2}$ They were found to agree with the results of these tests when the end fixation factor was taken into account and the effect of eccentricity noted. This curve, which goes over into the Euler hyperbola for large values of the slenderness ratio, has been plotted for comparison with the average results of this test.

In Figures 16 and 17 the dashed curves represent the Euler formula for round and for fixed ends, as shown. The dash-anddot curves shown represent the intermediate degree of end fixation for the Euler and their dotted continuation for the Kármán values that seemed to best fit the particular case.

Kármán made a special study of the effect of eccentric loading in column testing. It should be noted that the unit stresses he found are those from tests on 0.50 per cent carbon steel. The important feature is that a small eccentricity has the greatest effect in reducing the maximum unit load for values of slenderness ratio from 80 to 85 for round end columns $(f=I .0)$, and this is probably true for the milder steel in these angles. For other degrees of end fixation the critical slenderness ratio is obtained by multiplying, say, the value 85 by the value of $f$. It will be seen in the detailed discussion that the average value of the maximum unit load falls below the Kármán-Euler curves for these slenderness ratios, thus denoting the presence of eccentricity.

Since the lateral deflection at mid height of the columns was measured during the tests, it is possible to obtain a rough comparative measure at least of the eccentricity of the test specimens. For a column with fixed ends there can be no effective eccentric loading. The load, no matter how far its point of application is from the centroidal axis of the column, can only produce such stresses in a fixed end specimen as would be produced by a load concentrically applied. The reason for this is that the definition of a "fixed end" column presupposes that the tangent to the elastic curve at one end is parallel to and remains parallel to the tangent at the other end. In a testing machine, if the columns were really to have fixed ends, the bearing plates would remain 
parallel to each other throughout the test, and all effects of the eccentric loading would be taken up by the supporting screws of the testing machine. As a matter of fact, however, it is impossible to maintain this theoretical condition of fixed ends under an eccentric load either in a testing machine or in a built-up structure, and the column strength will be reduced if the load is applied eccentrically.

From the elastic theory one may express the relation between the lateral deflection at mid height, $\mathrm{y}_{\mathrm{m}}$, and the initial eccentricity, $y_{a}$, for round end columns, as follows:

$$
y_{\mathrm{m}}=y_{\mathrm{a}}\left(\frac{\mathrm{I}}{\cos \frac{l}{2 r} \sqrt{\frac{\sqrt{\mathrm{P}^{1}}}{a \mathrm{E}}}-\mathrm{I}}\right)
$$

where $P^{1}$ is the load which produced the deflection $y_{\mathrm{m}}$. Now, there is some value of $P^{1}=k P$ ( $P$ being the value of the maximum load from Euler's formula) for which the lateral deflection $y_{\mathrm{m}}$ is equal to the initial eccentricity $y_{\mathrm{a}}$. Solving for $k$ under this condition, $k=4 / 9$. That is, in a perfectly elastic round end column of any slenderness ratio the deflection at mid height when the load is $4 / 9$ of the theoretical maximum is equal to the initial eccentricity of load. For other degrees of end fixation this ratio would be different, but for the sake of comparison Tables 9 to i 2 show the lateral deflection at mid height which occurred at the unit loads $S^{1}=4 / 9 S$. It is assumed that the value of the theoretical unit load $S$ is given by the dash-and-dot curves of Figures 16 and I 7. Any other definite ratio might have been chosen for the comparison, provided the ratio were small enough, but the comparative results would have been practically the same. No claim is made that these values represent the actual initial eccentricity. It is, however, evident that in practically all cases the specimen of given slenderness ratio and degree of end fixation which sustained the highest unit load also suffered the least lateral deflection at the unit load $S^{1}$, while the specimen which suffered the greatest lateral deflection sustained the least unit load. Tables 9 to I2 also give the "rank" of the specimens according to strength and to lateral deflection. With very few exceptions the rank of a specimen is practically the same by either method of ranking.

One might conclude, then, from these results that the theoretical load-slenderness ratio curve for zero eccentricity should be drawn somewhat above the largest load values, and thus obtain a different value of fixation factor from the value obtained by considering the mean of the test results. It must, however, be pointed out 
that the specimens closely represented the conditions in actual construction, and no better centering of a member would be obtained on the average than was obtained in these tests. The mean results are therefore of more importance in design than any such theoretically determined values would be.

(b) Detailend Discussion of Results.-Figure i6 shows the results of tests of angles with square ends. The average result line is close to the fixed end curve (dotted), but agrees still better with the dash-and-dot curve plotted for $f=1.9$. At $l / r=200$ the average result is below the curve, and it is in this region that the most marked effects of eccentric loading are to be expected.

When the angles were tested with one bolt connection-in one leg only, approximately round end columns, - the results are close to the curve of end fixation, for $f=$ r.r. No appreciable effect of eccentricity in loading in reducing the maximum load appears here in the average results because the slenderness ratio is so much greater than 85 .

The results of tests of angles with folded ends, do not fall so close to the curve for $f=\mathrm{r}$. I as did those just considered. So few specimens of this class were tested that it is impossible to draw any definite conclusion. whether this type of column curve is suitable for angles with ends folded. It must also be noted that angles with ends folded, as shown in Figures 3, 6, and 8, have a variable radius of gyration from section to section. The ordinary column formulas are not derived for such conditions.

Figure 16 shows also the results of the tests of specimens held at each end with two bolts, in one leg only. This manner of fastening is more rigid than when a single bolt is used and the results for the columns with slenderness ratio as large as 200 lie close to the Euler curve for $f=\mathrm{I} .3$. For shorter columns the average results lie below this curve, and this may be due to the eccentric loading which would have the greatest effect at $l / r=$ r ro.

When two bolts are used, one in each leg, the degree of end fixation appears to be the same as for the previously considered class, and the results fall very close to the Euler curve for $f=\mathrm{r} .3$. The lengths tested in these two classes overlap for the slenderness ratios 200 and 250, and the average results for each of these slenderness ratios are nearly equal. The strength of the angles held with two bolts, in one leg only, is apparently the same as for specimens held with two bolts, one in each leg.

When two or more bolts were used in each leg for fastening the angle to the testing machine, the end fixation factor is still 
larger, and the curve for $f=\mathrm{I} .5$ of the Kármán-Euler type represents the average results very well, as shown in Figures $I 6$ and I7. Here, again, the eccentricity lowers the average result value at the critical slenderness ratio value, $85 \times \mathrm{I} .5=\mathrm{I} 27.5$, and is visible at $l / r=150$. When angles are held as rigidly as these were, it might have been expected that the end fixation factor would have been closer to the fixed end condition, $f=2.0$. It may be that the factor is no higher than I.5 because of the deformation which doubtless occurred in the structural members to which the test pieces were bolted.

\section{CONCLUSIONS.}

I. The values of the maximum unit load in these tests vary over a considerable range for any given slenderness ratio and manner of fastening the angles in the testing machine.

2. In most cases the specimen which sustained the greatest unit load for a given slenderness ratio and method of fastening suffered the least lateral deflection and the angle which bent most sustained the lowest unit load at failure, the deflection being measured at 4.9 of the theoretical maximum load.

3. For large slenderness ratios the average values are well represented by Euler's formula for long columns, calculated for different values of the end fixation factor.

4. The Kármán curves, recalculated for a yield point of 37,000 lbs./in. ${ }^{2}$ and modulus of elasticity of $30,000,000 \mathrm{lbs}$./in. ${ }^{2}$ represent the average results for small slenderness ratios for several methods of end fixation, except in the neighborhood of $l / r=80$ to 85 , where the effect of eccentricity was greatest. The values of the end fixation factor are given in Table 13 .

5. For angles with ends folded the column formulas considered do not represent the results found in this series of tests.

6. It is believed that these values of end fixation factor are of importance in the design of structures where the end conditions approximate those used in these tests, no matter what formula the designer prefers to use.

7. Eccentricity of loading produces a diminution of column strength. In these tests the greatest effect of eccentricity was observed in the neighborhood of a "free length" corresponding to $l / r=85$, which agrees with the results of Kármán's investigations.

Washington, April I2, 1922. 